\title{
La concentration des fonctions à haut contenu en savoir dans le secteur de la production des biens : quel avenir pour les régions non métropolitaines du Québec?
}

\section{The concentration of knowledge-rich functions in the goods producing sector: what future for non-metropolitan areas of Quebec?}

\section{La concentración de funciones de alto contenido en conocimientos en el sector de la producción de bienes: ¿Cual es el futuro de las regiones no metropolitanas de la provincia de Quebec?}

\section{Cédric Brunelle}

\section{Volume 56, numéro 158, septembre 2012}

Version originale soumise en novembre 2011. Version révisée reçue en juillet 2012.

URI : https://id.erudit.org/iderudit/1014549ar

DOI : https://doi.org/10.7202/1014549ar

Aller au sommaire du numéro

Éditeur(s)

Département de géographie de l’Université Laval

ISSN

0007-9766 (imprimé)

1708-8968 (numérique)

Découvrir la revue

Citer cet article

Brunelle, C. (2012). La concentration des fonctions à haut contenu en savoir dans le secteur de la production des biens : quel avenir pour les régions non métropolitaines du Québec ? Cahiers de géographie du Québec, 56(158), 313-342. https://doi.org/10.7202/1014549ar

\section{Résumé de l'article}

Les fonctions à haut contenu en savoir jouent un rôle stratégique au sein du secteur de la production des biens. Les régions et les entreprises où se concentrent ces fonctions semblent mieux positionnées que les autres pour assurer leur croissance et leur développement économique. Toutefois, cette réalité suggère de nouvelles disparités économiques à l'échelle régionale. Cet article analyse les tendances de concentration des fonctions à haut contenu en savoir dans les agglomérations du Québec entre 1971 et 2006. L’analyse soulève une concentration métropolitaine qui tend à s'accélérer durant la période. Néanmoins, on constate que certaines régions non métropolitaines ont des niveaux de croissance parfois supérieurs. S’il y a présence de trajectoires distinctes, on ne peut cependant conclure à la présence d'un processus de convergence régionale. Les résultats de l'analyse laissent entendre que la décentralisation de la production des biens a pu en grande partie se limiter aux fonctions de routine et de production. 


\section{La concentration des fonctions à haut contenu en savoir dans le secteur de la production des biens: quel avenir pour les régions non métropolitaines du Québec?}

The concentration of knowledge-rich functions in the goods producing sector: what future for non-metropolitan areas of Quebec?

La concentración de funciones de alto contenido en conocimientos en el sector de la producción de bienes: ¿Cual es el futuro de las regiones no metropolitanas de la provincia de Quebec?
Cédric BRUNELLE

Centre Urbanisation Culture Société

Institut national de la recherche scientifique Cedric.Brunelle@ucs.inrs.ca

\section{Résumé}

Les fonctions à haut contenu en savoir jouent un rôle stratégique au sein du secteur de la production des biens. Les régions et les entreprises où se concentrent ces fonctions semblent mieux positionnées que les autres pour assurer leur croissance et leur développement économique. Toutefois, cette réalité suggère de nouvelles disparités économiques à l'échelle régionale. Cet article analyse les tendances de concentration des fonctions à haut contenu en savoir dans les agglomérations du Québec entre 1971 et 2006. L'analyse soulève une concentration métropolitaine qui tend à s'accélérer durant la période. Néanmoins, on constate que certaines régions non métropolitaines ont des niveaux de croissance parfois supérieurs. S'il y a présence de trajectoires distinctes, on ne peut cependant conclure à la présence d'un processus de convergence régionale. Les résultats de l'analyse laissent entendre que la décentralisation de la production des biens a pu en grande partie se limiter aux fonctions de routine et de production.

\section{Mots-clés}

Fonctions, production des biens, professions, industries.

\section{Abstract}

Knowledge-rich functions play a strategic role within the goods producing sector. The regions and businesses where they cluster seem better positioned in terms of future growth and economic development. However, this process suggests new economic disparities at the regional level. Our article examines the concentration of knowledge-rich functions in Quebec urban areas between 1971 and 2006. We find patterns of increasing metropolitan concentration over this period. But higher growth levels can also be found in non-metropolitan areas. While several distinct regional trajectories can be observed, this does not mean we should conclude that a process of regional convergence is developing. Our results suggest that decentralization of production of goods may have largely been limited to routine and production functions.

\section{Keywords}

Functions, goods production, occupations, industry. 


\section{Resumen}

Las funciones a alto contenido de conocimientos juegan un rol estratégico dentro del sector de producción de bienes. Las regiones y empresas donde se concentran esas funciones parecen posicionarse mejor que las otras, asegurando así su crecimiento y desarrollo económico. Sin embargo, esta realidad sugiere nuevas desigualdades económicas a escala regional. Este artículo analiza las tendencias de concentración de esas funciones en las aglomeraciones de la provincia de Quebec entre los años 1971 y 2006. El análisis señala una concentración metropolitana que tiende a acelerarse durante este período. Sin embargo, se constata que ciertas regiones no metropolitanas a veces tienen niveles de crecimiento superiores. Bien que haya trayectorias diferentes, no se puede concluir sobre la presencia de procesos de convergencia regional. Los resultados del análisis dejan entender que le descentralización de la producción de bienes ha podido, en gran parte, limitarse a las funciones de rutina y de producción.

\section{Palabras claves}

Funciones, producción de bienes, profesiones, industrias.

\section{Introduction}

L’ouverture économique des marchés et le changement technologique accéléré des 15 dernières années ont eu de nombreux impacts sur la structure de l'emploi manufacturier au Québec. L’exemple de Bombardier n'en est qu'un parmi plusieurs ${ }^{1}$. Les restructurations, les délocalisations et les impartitions font aujourd'hui partie intégrante des stratégies d'adaptation des entreprises au contexte économique global.

La tendance générale, soulevée dans des travaux aux États-Unis, en Europe et au Canada, est celle d'une division géographique croissante entre les fonctions à haut contenu en savoir et les fonctions routinières et de production au sein des entreprises (Bade et al., 2004 ; Duranton et Puga, 2005 ; Brunelle et Polèse, 2008; Hendricks, 2011). L'exemple de la division spatiale entre siège social - localisé dans une métropole et site de production - localisé dans un centre urbain secondaire - reste classique dans la littérature (Pred, 1974 et 1975 ; Westaway, 1974 ; Massey, 1984).

D’autres schémas ont depuis émergé, élargissant cette logique à un vaste ensemble d'activités. Les auteurs notent une décomposition géographique de plus en plus fine des segments fonctionnels de l'entreprise - gestion stratégique, recherche et développement, marketing, approvisionnement, centres de services, logistique, service après vente, productions intermédiaires, montage, etc. - accompagnée d'une reconfiguration

1 Récemment, Bombardier rendait publique son intention de transférer le service d'ingénierie de son usine de La Pocatière, au Bas-Saint-Laurent, vers ses installations de Saint-Bruno-de-Montarville, en banlieue de Montréal. Au total, près de 50 ingénieurs sur les 300 employés de l'usine seraient délocalisés vers la métropole (Radio-Canada, 2011). La région, déjà aux prises avec une baisse des effectifs de l'usine, ferait maintenant face à la possibilité de ne concentrer que les segments montage et production de la chaîne d'opération de la multinationale. 
spatiale favorisant la concentration de fonctions similaires dans de mêmes types de régions (Sturgeon et Gereffi, 2008 et 2009; Baldwin, 2009). En outre, la mobilité induite par les nouvelles technologies et l'ouverture des marchés générerait une spécialisation économique croissante des régions le long de la chaîne verticale de production (Hummels et al., 2001 ; Defever, 2005 et 2006; Duranton et Puga, 2005 ; Dean et al., 2007 ; Lobo e Silva et Hewings, 2010).

Ces transformations ne sont pas neutres pour les trajectoires régionales de développement économique. L'économie du savoir implique qu'une part croissante de la valeur ajoutée dans la production des biens provienne de segments à plus fort contenu en services. L'accélération du cycle des produits et l'interpénétration mondiale des marchés font surgir la nécessité du positionnement et de l'adaptabilité des produits à un contexte en croissante évolution. La recherche et le développement (R\&D), le design, la gestion et le marketing sont aujourd'hui des fonctions stratégiques de premier ordre pour l'industrie. Les régions et les entreprises où se concentrent ces fonctions seraient non seulement avantagées sur le plan de leur capital humain et de leur productivité, mais elles posséderaient un plus fort potentiel de croissance et de développement économique (OCDE, 2007 ; Sturgeon et Gereffi, 2009; Moretti, 2010) ${ }^{2}$. En contrepartie, la standardisation des procédés de production et la «routinisation» de plusieurs fonctions intermédiaires ont eu tendance à décroître la valeur relative d'activités à plus faible contenu en savoir. On observe que ces fonctions sont aussi sujettes à des délocalisations plus fréquentes - la standardisation favorisant la simplification des procédés et une implantation facilitée dans de nouveaux contextes géographiques (Leamer et Storper, 2001). Les régions spécialisées dans les fonctions routinières et de production seraient ainsi plus vulnérables aux aléas de la conjoncture économique.

Cet article propose d'analyser la division spatiale des fonctions dans le secteur de la production des biens au Québec. Cinq sous-secteurs sont examinés : extraction primaire et première transformation, ainsi que production de basse, de moyenne et de haute technologie. L'étude vise à dégager les grandes tendances, dans l'ensemble et pour chaque sous-secteur, de la concentration régionale des travailleurs à haut contenu en savoir relativement aux fonctions de routine et de production, de 1971 à 2006. Sur un total de 134 villes canadiennes comparables durant cette période, notre base de données comprend 29 agglomérations urbaines et 7 régions synthétiques au Québec.

L'article aborde deux questions : assiste-t-on à une fragmentation régionale généralisée entre les fonctions à haut contenu en savoir et les fonctions plus routinières dans le secteur de la production des biens? Quel avenir se dessine pour le développement des fonctions à haut contenu en savoir dans les régions non métropolitaines? Ces questions représentent un enjeu central pour l'élaboration de stratégies de développement économique régional. Une division géographique croissante entre les fonctions à haut contenu en savoir et les fonctions de routine et de production laisse présager, dans le contexte de l'économie du savoir, le spectre d'un développement régional de plus en plus asymétrique.

2 Outre leur aspect stratégique au sein des réseaux productifs, les fonctions à haut contenu en savoir ont de nombreux impacts sur le marché local de l'emploi. Le plus direct tient à l'effet du multiplicateur régional. Moretti (2010) estime que chaque travailleur additionnel dans le secteur manufacturier résulte en une création indirecte de 1,6 emploi dans les biens et services locaux. En comparaison, les niveaux de salaire plus élevés des travailleurs qualifiés font passer à 2,5 le nombre d'emplois locaux générés par travailleur qualifié supplémentaire. 


\section{Transformation du secteur de la production des biens}

\section{Le rôle croissant des fonctions à haut contenu en savoir}

Au cours des 25 dernières années, la production des biens a connu une suite de restructurations ponctuées par les fermetures d'usines et les délocalisations. En conséquence, le poids relatif du secteur dans l'économie des pays avancés n’a cessé de diminuer ${ }^{3}$. Le Québec n’y fait pas exception. Entre 1971 et 2006, l'emploi dans la production des biens est passé de près de $32 \%$ à moins de $19 \%$ du total de la province (annexe 1). Ce déclin relatif et la montée simultanée des activités tertiaires dans l'économie sont en partie tributaires du déplacement international de la production vers les pays émergents comme l'Inde et la Chine, plus compétitifs sur le plan des salaires. Toutefois, les secteurs des services, particulièrement ceux à plus forte intensité en connaissance, n'ont pour la plupart pas suivi.

Paradoxalement, on observe une croissance analogue des activités tertiaires dans la production des biens, qui tend à mobiliser une part de plus en plus importante de services spécialisés intrabranches. Le secteur serait donc moins une activité en déclin stricto sensu qu'une industrie dont la restructuration accélérée favorise la rétention et la croissance de fonctions spécialisées. Au Québec, alors que le secteur connaissait un taux de croissance annuel moyen de 0,3\% entre 1991 et 2006 - largement inférieur au taux de 1,9\% pour l'économie de la province dans son ensemble -, les fonctions à haut contenu en savoir à l'intérieur du secteur affichaient une croissance annuelle moyenne de 3,1 \% (annexe 1). Quoique légèrement inférieurs, ces taux restent comparables à la moyenne canadienne ${ }^{4}$.

Ces tendances soulèvent une distinction importante si l'on considère que les segments à haut contenu en savoir sont ceux qui contribuent aujourd'hui le plus à la valeur ajoutée des produits. Avec le commerce international et l'automatisation des procédés, la fabrication d'un objet physique, qu'il s'agisse d'un téléphone intelligent, d'un comprimé pharmaceutique ou d'un vêtement dernier cri, ne représente plus qu'une infime part du coût total entourant sa commercialisation. La principale partie de la valeur, encapsulée dans l'objet physique, provient désormais de fonctions spécialisées: recherche et développement, design, finance, propriété intellectuelle, marketing, gestion stratégique, etc. Il ne s'agit pas d'affirmer que les segments traditionnels ne sont plus des activités intéressantes pour les firmes et les régions, mais de reconnaître que les productions qui gagnent davantage de valeur sont aujourd'hui celles qui répondent et s'adaptent le plus rapidement au marché.

\section{La concentration métropolitaine des fonctions à haut contenu en savoir}

Le rôle croissant des fonctions à haut contenu en savoir dans la production des biens ne profite pas à toutes les régions de la même manière. D'un point de vue de l'économie spatiale, les travaux récents suggèrent que la fragmentation géographique des

3 Pour une définition du secteur, voir l'annexe 2.

4 Au Canada, la croissance annuelle moyenne de l'emploi sur la période est de 1,5\%, de 0,3\% pour le secteur de la production des biens, et de $2,8 \%$ pour les fonctions à haut contenu en savoir dans le secteur de la production des biens. 
fonctions intrabranches a pu entraîner une spécialisation fonctionnelle des villes et régions (Bade et al., 2004; Defever, 2005 et 2006; Duranton et Puga, 2005; Brunelle et Polèse, 2008; Rossi-Hansberg et al., 2009; Lobo e Silva et Hewings, 2010). Plusieurs concepts y font référence directement ou implicitement dans la littérature: séparation entre siège social et production (Chandler, 1977; Duranton et Puga, 2005; Fujita et Thisse, 2006; Aarland et al., 2007; Davis et Henderson, 2008), fragmentation spatiale de la chaîne de valeur (Defever, 2005 et 2006; Sturgeon et Gereffi, 2008 et 2009), grappes socioprofessionnelles (Thompson, 1986; Koo, 2005; Barbour et Markusen, 2007), spécialisation verticale (Hummels et al., 2001; Dean et al., 2007) ou sous-traitance des fonctions d'entreprises (Ono, 2003; Helsley et Strange, 2007; Huws et al., 2009; Sturgeon et Gereffi, 2009). Les fonctions manuelles et de routine (assemblage, traitement de données, télémarketing, etc.) tendent à se déplacer vers des localités plus petites et à moindres coûts, alors que les fonctions à forte intensité en connaissances - sous influence des économies d'agglomération - se centraliseraient progressivement dans les grandes zones urbaines (Henderson et al., 2001). Comme elles sont adoptées par un ensemble de firmes, ces dynamiques se renforcent mutuellement, transformant la structure du marché de l'emploi des villes relativement à leur position dans les systèmes urbains.

\section{Hypothèses pour le Québec}

Malgré le peu d'études de cas au Québec, le contexte récent laisse présager que le phénomène ait pu s'accentuer au cours des 15 dernières années. La déconcentration manufacturière des grands centres au profit des régions centrales du sud-est, mieux positionnées pour l'exportation, a très certainement été accélérée par l'entrée en vigueur de l'Accord de libre-échange nord-américain (ALENA) en 1994. Les auteurs parlent de l'émergence d'un «arc industriel» (Polèse, 2009) ou d'un «croissant manufacturier» (Proulx, 2009) pour décrire une forte localisation d'activités industrielles sur un territoire se déployant de la Montérégie au Bas-Saint-Laurent, en passant par le Centre-du-Québec et la Beauce. Cette nouvelle tendance contraste avec la concentration historique du secteur dans la région métropolitaine avant les années 1980 alors que Montréal représentait un centre manufacturier d'importance à l'échelle québécoise et canadienne. Notre hypothèse est que cette déconcentration des activités industrielles hors des grands centres urbains du Québec aurait été alimentée par une fragmentation fonctionnelle croissante des activités du secteur. Pour l'arc industriel et les régions périphériques de la province, nous croyons donc que la déconcentration de l'emploi se serait limitée à des fonctions routinières et de production, s'y localisant pour tirer avantage des coûts inférieurs. L'hypothèse corollaire est que les agglomérations métropolitaines de la province - en particulier Montréal et Québec - auraient renforcé leur position pour les segments à plus forte intensité en savoir, favorisant leur émergence comme centres de services spécialisés pour l'industrie de la fabrication. Dans les deux cas, l'hypothèse sous-tend un développement de plus en plus asymétrique des régions de la province. Les régions non métropolitaines s'orienteraient vers un affaiblissement de leurs fonctions à plus forte intensité en connaissance, alors que les grands centres urbains continueraient de concentrer les activités à plus forte valeur ajoutée. Les paragraphes suivants visent à évaluer cette hypothèse en regard de l'hypothèse inverse d'une convergence régionale durant la période. 


\section{Données utilisées}

Les données utilisées sont tirées de six recensements du Canada (1971, 1981, 1991, 1996, 2001, 2006), basées sur des tabulations spéciales de Statistique Canada. La série complète couvre l'ensemble du Canada, avec l'emploi pour unité d'analyse. Afin d'assurer une comparabilité temporelle, l'étude se concentre principalement sur les zones urbaines, définies comme l'ensemble des lieux urbains avec une population d'au moins 10000 habitants en $2006^{5}$. Exception faite des analyses cartographiques pour 2006, où elles sont représentées au niveau des divisions de recensement (DR), les zones rurales sont exclues de la plupart des étapes de l'analyse, car des changements de limites en 2001 rendent impossibles les comparaisons directes entre les unités spatiales temporelles. Au total, notre base de données comprend 134 observations en milieu urbain (unités spatiales), qui représentaient $81 \%$ de l'emploi au Canada en 2006. Pour le Québec, la base inclut 29 agglomérations comparables sur la période. Les villes sont soit des agglomérations de recensement (AR), ou des régions métropolitaines de recensement (RMR) qui sont des bassins d'emploi distincts délimités par les flux de navettage, tels que définis par Statistique Canada. À cet égard, seule la RMR de Gatineau nous a posé un problème de par sa forte intégration économique avec celle d'Ottawa. Comme il ne nous était pas possible de départager les deux bassins d'emploi, nous avons choisi de l'exclure de l'analyse ${ }^{6}$.

Le principal problème conceptuel de notre étude tient à la définition du concept «fonction». Sauf dans les études de cas, mesurer directement les fonctions (ensembles de tâches et de compétences) est rarement possible. Par ailleurs, "fonction» n'est pas un concept statistique utilisé dans le recensement canadien. Dans la littérature, l'équivalent le plus couramment utilisé est celui de "profession", où les catégories socioprofessionnelles sont agrégées pour délimiter les fonctions internes de l'entreprise, partant de Thompson et Thompson (1987) et la nomenclature professionnellefonctionnelle jusqu'aux travaux plus récents sur la fragmentation spatiale de la chaîne de valeur et l'externalisation des fonctions d'entreprises (Brown, 2008; Huws et al., 2009; Sturgeon et Gereffi, 2009). Une approche similaire, reposant sur la Classification nationale des professions - statistiques (CNP-S) de 2001 et de la Classification type des professions (CTP) de 1981-1991, est adoptée ici. Pour prendre en compte l'évolution de la définition des catégories professionnelles au fil du temps, il nous a été nécessaire de construire une concordance entre les différents systèmes de classification pour assurer la comparabilité longitudinale. Les catégories professionnelles ont été créées suivant les lignes directrices prescrites par Statistique Canada (2002), résultant en six classes agrégées (Statistique Canada, CNP-S 2001, codes entre parenthèses) :

1. Gestion, administrateurs et personnel assimilé (A, B0, B1, B3)

2. Sciences naturelles et sociales, mathématiques, ingénierie (C, E0, E211, E212, E213)

5 Ces zones incluent les agglomérations de recensement (AR) et les régions métropolitaines de recensement (RMR), telles que définies par Statistique Canada.

6 Notre base de données comprend des données au lieu de résidence et non au lieu de travail, ce qui nécessite l'agrégation spatiale à de plus larges échelles (RMR, AR et SDR de plus de 10000 habitants) afin d'éviter les problèmes liés au navettage. La RMR de Gatineau, où plus de la moitié des travailleurs font la navette quotidienne vers Ottawa, est donc problématique lorsqu'elle est séparée de la RMR d'Ottawa. Elle n'a donc pas été incluse dans notre analyse. 
3. Éducation (enseignants, formateurs), travailleurs de la santé, artistes, clergé et personnel assimilé (D, E1, E214, E215, E216, F)

4. Personnel de bureau et services connexes (B2, B4, B5)

5. Vente et services $(G)$

6. Production et entretien: ouvriers de production, métiers, opérateurs, mines, agriculture, exploitation forestière, construction (H, I, J)

Bien que ces grands agrégats socioprofessionnels ne soient pas optimaux pour délimiter les catégories de compétences, l'utilisation de tabulations croisées (entre catégories professionnelles et industrie) laisse peu de choix si nous souhaitons conserver un nombre suffisant de cas (emplois) par unité spatiale. Ainsi, les six classes sont réduites à deux fonctions - haut contenu en savoir/routine-production - dans un second temps (annexe 2). Nous pouvons raisonnablement supposer que les deux premiers groupes professionnels sont un bon indicateur de fonctions riches en connaissances, valables dans tous les secteurs. Les deux premiers groupes professionnels ont à la fois de plus fortes proportions de diplômés universitaires et des revenus plus élevés que les trois derniers (annexe 2), indiquant une plus grande sensibilité aux économies d'agglomération. Lorsque cela est possible, la fonction haut contenu en savoir, qui inclut les classes 1 (gestion) et 2 (scientifiques-professionnels), est présentée de façon désagrégée. La fonction routine-production inclut quant à elle les classes 4 (soutien administratif), 5 (vente) et 6 (production). La classe éducation a été retirée de l'analyse, correspondant à un ensemble résiduel de travailleurs difficile à classifier dans la typologie retenue. En termes de niveaux de capital humain et de salaires, la différence des classes nous semble appréciable. Pour la classe haut contenu en savoir en 2006, 40,8\% des travailleurs ont un niveau d'éducation supérieur au baccalauréat et un salaire annuel moyen de 69435 , alors que les chiffres correspondants sont de $15,7 \%$ et $41662 \$$ pour la fonction routine-production (annexe 2).

Le secteur de la production des biens regroupe également plusieurs industries. L'ensemble des données de base, avec des définitions industrielles standardisées entre 1971 et 2006, contenait 126 classes d'industries. Ces dernières ont été agrégées en cinq industries pour le secteur de la production des biens, soit quatre sous-secteurs manufacturiers et un secteur incluant l'ensemble des industries liées à l'extraction primaire (annexe 3). La production des biens exclut en somme l'ensemble des secteurs de services de l'économie. Le choix d'agrégation en cinq industries se justifie par la difficulté de conserver des valeurs avec des niveaux acceptables pour les 134 unités spatiales, compte tenu de l'échantillonnage et des méthodes d'arrondissement de Statistique Canada. Selon l'erreur type, les valeurs en dessous de 50 peuvent varier de $30 \%$ dans un sens ou dans l'autre, ce qui est amplifié lorsqu'on retourne aux périodes initiales de notre base de données. Pour certaines statistiques descriptives, les zones urbaines sont regroupées en sept classes composites (régions synthétiques), qui sont cohérentes dans le temps (annexe 4), basées sur des critères de taille et de distance, adaptées de Brunelle et Polèse (2008) et Polèse et Shearmur (2006) ${ }^{7}$.

7 L'auteur reconnaît l'existence du Modifiable areal unit problem (MAUP), qui soulève le problème potentiel d'une hétérogénéité interne à la typologie. Pour offrir davantage de robustesse, des analyses désagrégées par villes sont proposées dans les sections subséquentes de l'article. La typologie reste néanmoins statistiquement valide pour décrire les dynamiques à l'échelle macrorégionale: des analyses de variance (ANOVA) réalisées sur les données désagrégées ont révélé des différences significatives entre les classes pour l'ensemble des variables présentées dans cet article. 
Pour mesurer la concentration régionale des fonctions, l'indicateur retenu repose sur celui proposé par Duranton et Puga (2005) qui, à partir de la moyenne du système, calcule les déviations à la moyenne (DM) des cadres et des gestionnaires par travailleur de production dans le secteur manufacturier ${ }^{8}$. Notre approche se distingue par l'inclusion des fonctions routinières (support administratif, vente et services) à la classe production spécifiée dans leur étude, afin de rendre compte d'une division potentielle entre les fonctions de services supérieurs et les fonctions routinières. Malgré sa forte similitude arithmétique avec le quotient de localisation (QL), la DM s'est révélée être l'indicateur le plus approprié dans la mesure de la spécialisation fonctionnelle. Contrairement au QL, dont la distribution peut varier entre zéro et l'infini, la DM varie entre -1 et 1 . Dans le cas du QL, cette caractéristique implique que des changements importants au niveau du dénominateur rendent deux distributions incomparables entre elles. Or, la croissance fulgurante des fonctions à haut contenu en savoir dans l'économie québécoise a précisément cet effet sur les distributions entre 1971 et 2006. En conservant un intervalle constant à chaque période, la DM se soustrait à ce problème et permet d'éviter des transformations complexes dans les distributions.

Notre étude procède en trois étapes, débutant par une analyse descriptive des tendances générales de concentration dans le système urbain québécois. Dans un deuxième temps, l'analyse met en lumière certaines trajectoires régionales, notamment par l'analyse des niveaux de croissance au cours de la période. Enfin, l'article se termine par une série comparative de régressions testant l'hypothèse de la convergence des régions au Québec entre 1971 et 2006.

\section{La concentration des fonctions scientifiques et de gestion dans le système urbain québécois}

\section{Évolution dans cinq sous-secteurs: vers une concentration métropolitaine}

Dans son ensemble, la tendance est vers une concentration métropolitaine des fonctions à haut contenu en savoir. Alors que ces fonctions représentaient 19,5\% de l'emploi total du secteur de production des biens dans la province en 2006, elles atteignaient plus de $27 \%$ à Montréal et plus de $23 \%$ à Québec, comptant pour moins de $13 \%$ dans les régions centrales et périphériques de moins de 100000 habitants (annexe 1).

La figure 1 permet de creuser davantage ces tendances. Elle présente schématiquement les courbes de déviation de la moyenne ${ }^{9}$ des fonctions de gestion (figure 1a) et des fonctions scientifiques/professionnelles (figure 1b) pour chacun des cinq sous-secteurs, dans sept grandes régions du système urbain québécois, pour les périodes de 1971, 1991 et 2006. L'interprétation est assez directe: les valeurs au-dessus de zéro indiquent une déviation positive relativement à la moyenne québécoise, alors que les valeurs inférieures montrent une déviation négative. Les régions sont ordonnées sur l'axe des abscisses afin de représenter la hiérarchie urbaine québécoise et la distance aux grands centres: une courbe descendante de gauche à droite nous indique un schéma de localisation hiérarchique où les activités tendent vers une concentration métropolitaine, alors qu'une courbe ascendante nous présente une concentration hors des métropoles, dans les régions éloignées.

8 Équation [002] de l'annexe 5.

9 Équation [002] de l'annexe 5. 
Figure 1 Gestionnaires et scientifiques/professionnels par classes industrielles. Agglomérations de recensement et régions synthétiques. Déviations de la moyenne québécoise, 1971, 1991 et 2006

a) Gestion

Extraction primaire

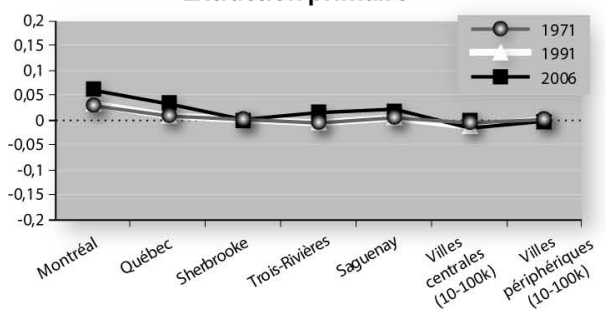

Première transformation

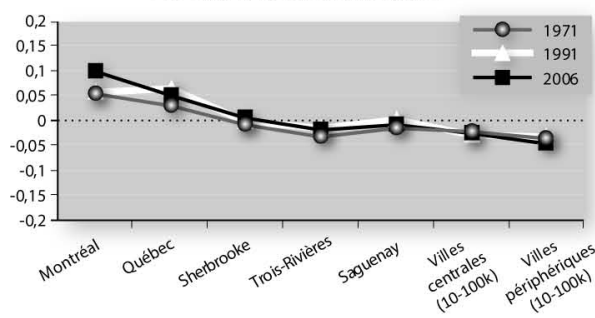

Manufacturiers de basse technologie

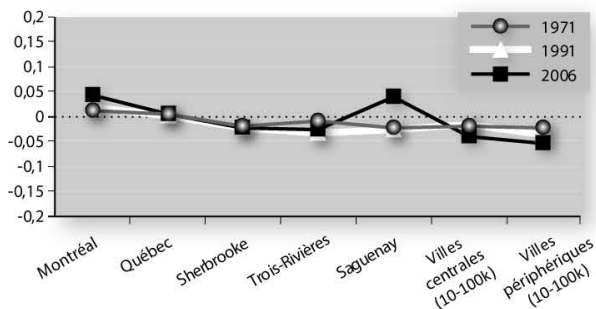

Manufacturiers de moyenne technologie

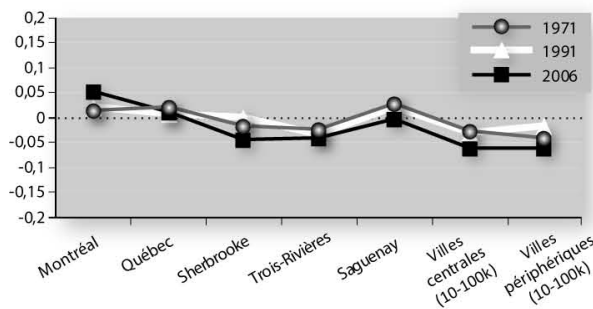

Manufacturiers de haute technologie

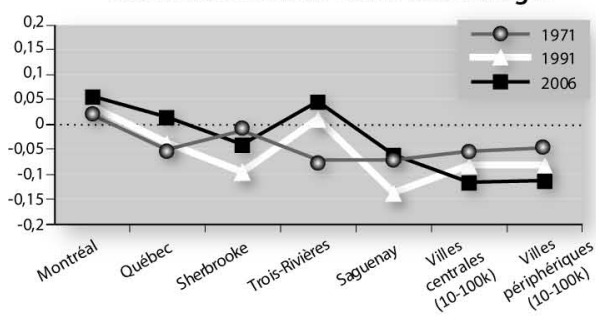

b) Scientifiques/Professionnels Extraction primaire

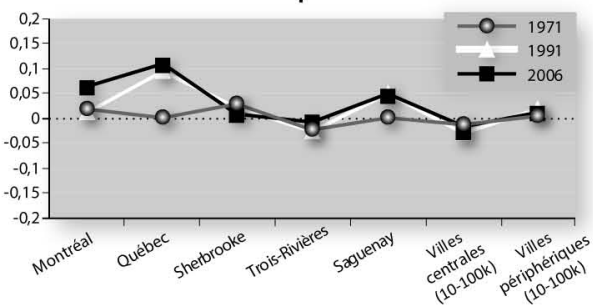

Première transformation

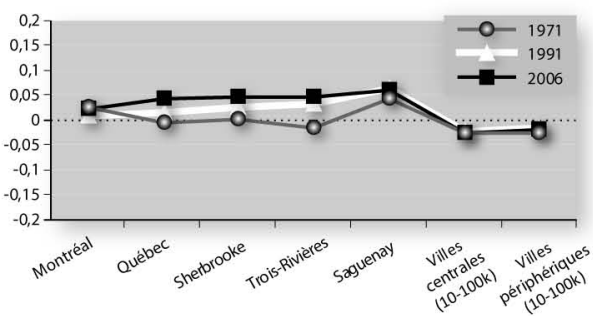

Manufacturiers de basse technologie

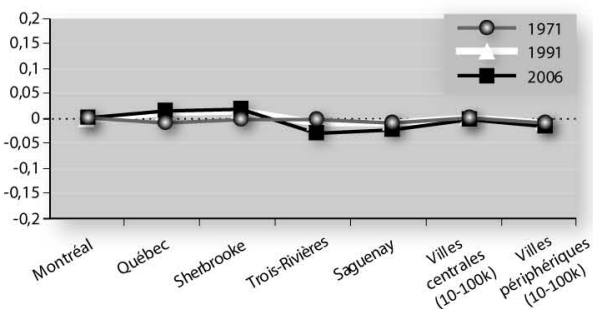

Manufacturiers de moyenne technologie

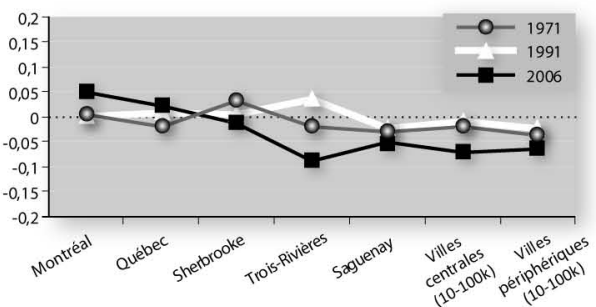

Manufacturiers de haute technologie

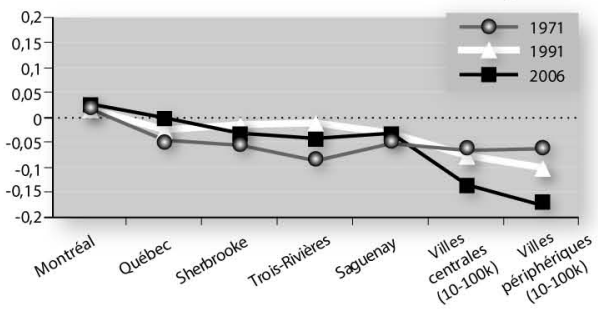


Les schémas nous montrent que la fonction de gestion suit une localisation hiérarchique croissante dans les cinq sous-secteurs de la production des biens (figure 1a). Pour les RMR de Montréal et Québec, la proportion locale de gestionnaires tend à se distancer de la moyenne québécoise sur les trois périodes - tendance nettement plus marquée à Montréal après 1991. Le phénomène corollaire est une déviation négative de plus en plus importante dans les régions non métropolitaines, plus particulièrement dans les villes centrales et périphériques de moins de 100000 habitants. Les cas les plus frappants se trouvent dans les secteurs d'extraction primaire et de première transformation, qui incluent une bonne part d'emploi dans les industries forestières, minières et agricoles. Presque par définition, toutes les fonctions de production sont exclues des grands centres urbains. Les travailleurs forestiers, les foreurs ou les agriculteurs ne sont pas des professions communes dans les grandes villes. Le résultat inévitable est une séparation spatiale entre les professions de gestion du secteur, concentrées dans les grandes villes, et les fonctions de production ailleurs - ce constat tenant aussi pour le secteur de la première transformation.

Les secteurs manufacturiers de basse, moyenne et haute technologie offrent un schéma hiérarchique similaire, où la gestion se concentre quasi exclusivement et de façon croissante dans les RMR de Montréal et Québec, et de façon plus marquée après 1991 (figure 1a). La fonction n'a, semble-t-il, pas suivi la déconcentration manufacturière massive des 25 dernières années, tirant vraisemblablement profit d'une localisation métropolitaine stratégique. Seuls cas divergents en 2006, Trois-Rivières se dirige vers une concentration de la fonction de gestion pour le secteur de la haute technologie, alors que Saguenay concentre ces fonctions pour le manufacturier de basse technologie. Pour Trois-Rivières, cette tendance peut en partie s'expliquer par la localisation intermédiaire de la région entre les centres décisionnels de Québec et Montréal - une situation quasi unique au Québec pour une ville universitaire. Le cas de Saguenay semble, quant à lui, davantage conjoncturel qu'une tendance émergente - ce qui reste à confirmer dans les années futures -, les déviations positives pour les fonctions de gestion résultant de pertes massives d'emploi pour les fonctions de routine-production entre 2001 et 2006. Pour le reste, la gestion demeure concentrée à Montréal et Québec.

De façon similaire, les fonctions scientifiques et professionnelles montrent une localisation hiérarchique dans le système urbain québécois, ce que nous observons dans le manufacturier de moyenne et de haute technologie, où les tendances sont nettement plus marquées après 2006 (figure 1b). On constate toutefois que leur distribution spatiale y est beaucoup plus hétérogène que dans les autres secteurs. Exception faite du manufacturier de basse technologie à Trois-Rivières, elles se concentrent à Québec, Sherbrooke, Trois-Rivières et Saguenay. Cette plus forte présence régionale souligne une différence sectorielle dans le potentiel de division spatiale des fonctions. Ainsi, bien que les technologies permettent de fragmenter un nombre croissant de fonctions spécialisées du processus de production, on constate que plusieurs activités continuent de nécessiter la présence locale de travailleurs spécialisés. Pour ces dernières, une localisation intermédiaire entre les nombreux sites de production exploités dans un rayon de proximité peut s'avérer un facteur positif sur la colocalisation des fonctions scientifiques et de production. Dans d'autres cas, cette colocalisation peut être accentuée par la structure même de l'entreprise, où les secteurs dominés par les entreprises de moyenne taille sont, relativement aux secteurs dominés par les grandes entreprises multiétablissements, généralement plus intégrés spatialement. Ces facteurs peuvent en 
partie expliquer la fragmentation plus importante des fonctions scientifiques dans les secteurs d'extraction, de moyenne et de haute technologie, relativement aux secteurs de première transformation et manufacturiers de basse technologie.

\section{Dimension géographique: présence de pôles régionaux de services intrabranches}

Pour les données désagrégées, la cartographie du quotient de localisation (figures 2a et 3a), décrivant la concentration des secteurs relativement à la moyenne canadienne, ainsi que celle de la spécialisation fonctionnelle pour la gestion (figures $2 b$ et $3 b$ ) et les fonctions scientifiques (figures $2 \mathrm{c}$ et $3 \mathrm{c}$ ) témoignent de la présence de pôles régionaux de services à l'intérieur de ces industries.

Pour les extractions primaires, on observe que les fonctions à haut contenu en savoir se concentrent dans un nombre limité de centres régionaux, dont Sept-Îles, Saguenay, Val-d'Or, Rouyn-Noranda, Rivière-du-Loup et Saint-Hyacinthe (figure 2b, c). Alors que le secteur dans son ensemble ne représente qu'une faible part de l'emploi total, la spécialisation fonctionnelle de ces villes contraste avec la forte concentration du secteur dans les régions qui leur sont limitrophes (figure 2a). Représentées par des flèches, ces relations spatiales entre les centres régionaux où se concentrent les fonctions scientifiques et de gestion et la localisation du secteur dans son ensemble témoignent d'une articulation spatiale classique entre un centre et son hinterland. L'exploitation des ressources naturelles dans la périphérie et dans les zones rurales aurait, semble-t-il, favorisé une structure spatiale où les activités à plus fort contenu en services tendent à se polariser vers les centres régionaux à proximité.

Quoiqu'à une échelle spatiale plus restreinte, ces mêmes dynamiques sont aussi présentes dans le secteur manufacturier - le secteur de basse technologie offre un bon exemple des tendances générales du secteur (figure 3a, b et c). Ainsi, malgré le fait que la fonction de gestion se concentre quasi exclusivement à Montréal, on observe la présence de plusieurs centres régionaux secondaires pour les fonctions scientifiques, soit à Saint-Georges, Drummondville, Victoriaville, Saint-Hyacinthe et Sherbrooke ${ }^{10}$. Ces résultats indiquent que, pour le secteur manufacturier, la fonction de gestion tend vers une plus grande fragmentation géographique que la fonction scientifique - la colocalisation avec la production favorisant leur concentration dans les agglomérations urbaines régionales.

Ensemble, les deux secteurs cartographiés montrent que les fonctions possèdent une géographie distincte de l'ensemble des activités du secteur. Les résultats révèlent l'existence de pôles de services régionaux intrabranches. Malgré une forte concentration métropolitaine, ces tendances suggèrent un continuum plus hétérogène qu'il n’y paraît de prime abord. Cette piste est explorée dans les paragraphes suivants.

10 Des tendances similaires sont observées dans les autres secteurs manufacturiers non cartographiés. 
Figure 2 (a) Spécialisation dans le secteur de l'extraction; spécialisation dans les fonctions de (b) gestion et (c) professionnelles et scientifiques, Québec 2006
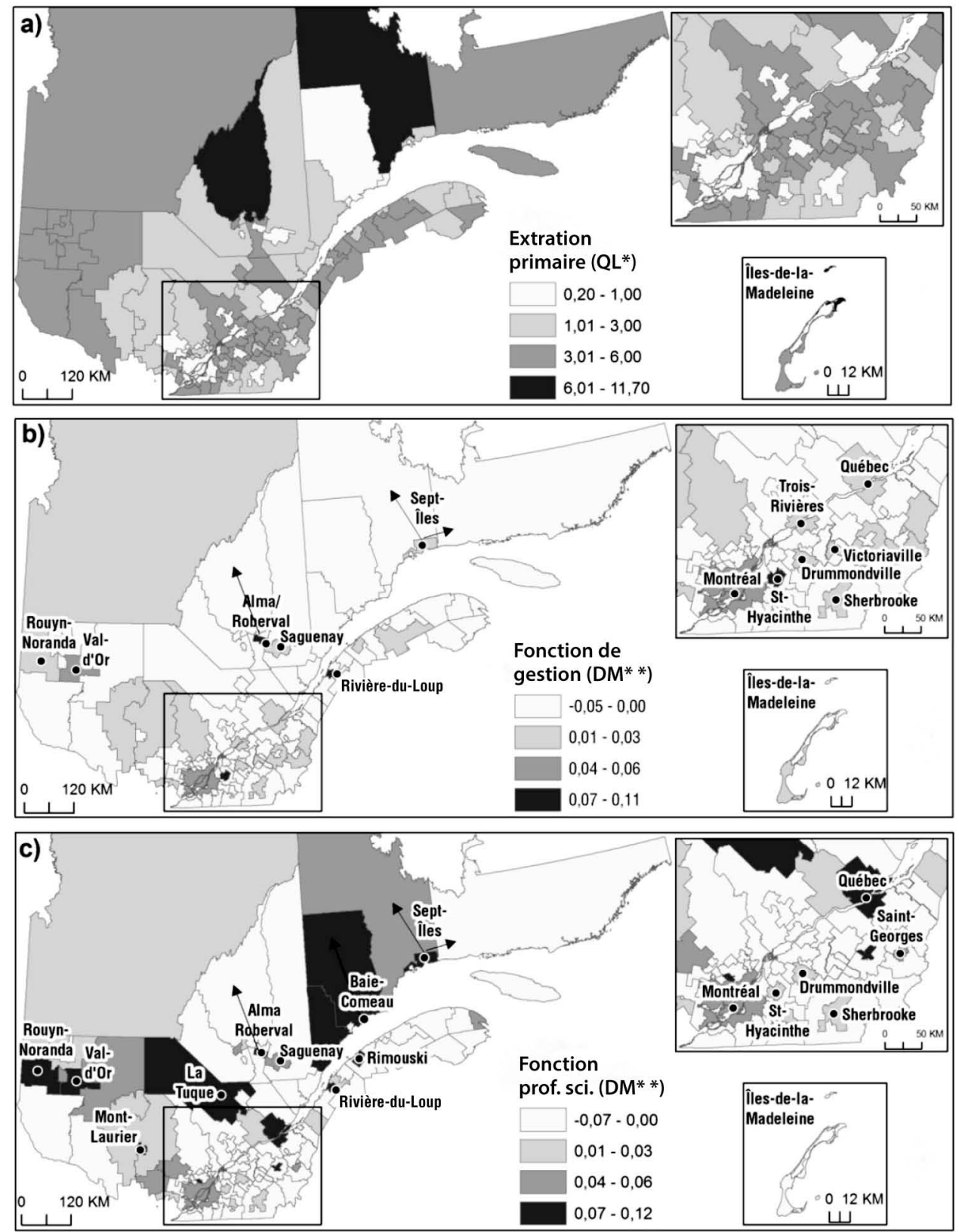

Source: Statistique Canada, Recensement de 2006; *QL - Quotient de localisation relatif à la moyenne canadienne; ${ }^{*}$ DM - Déviations de la moyenne québécoise. Cartographie Cédric Brunelle, 2012. 
Figure 3 (a) Spécialisation dans le secteur manufacturier de basse technologie; spécialisation dans les fonctions de (b) gestion et (c) professionnelles et scientifiques, Québec 2006
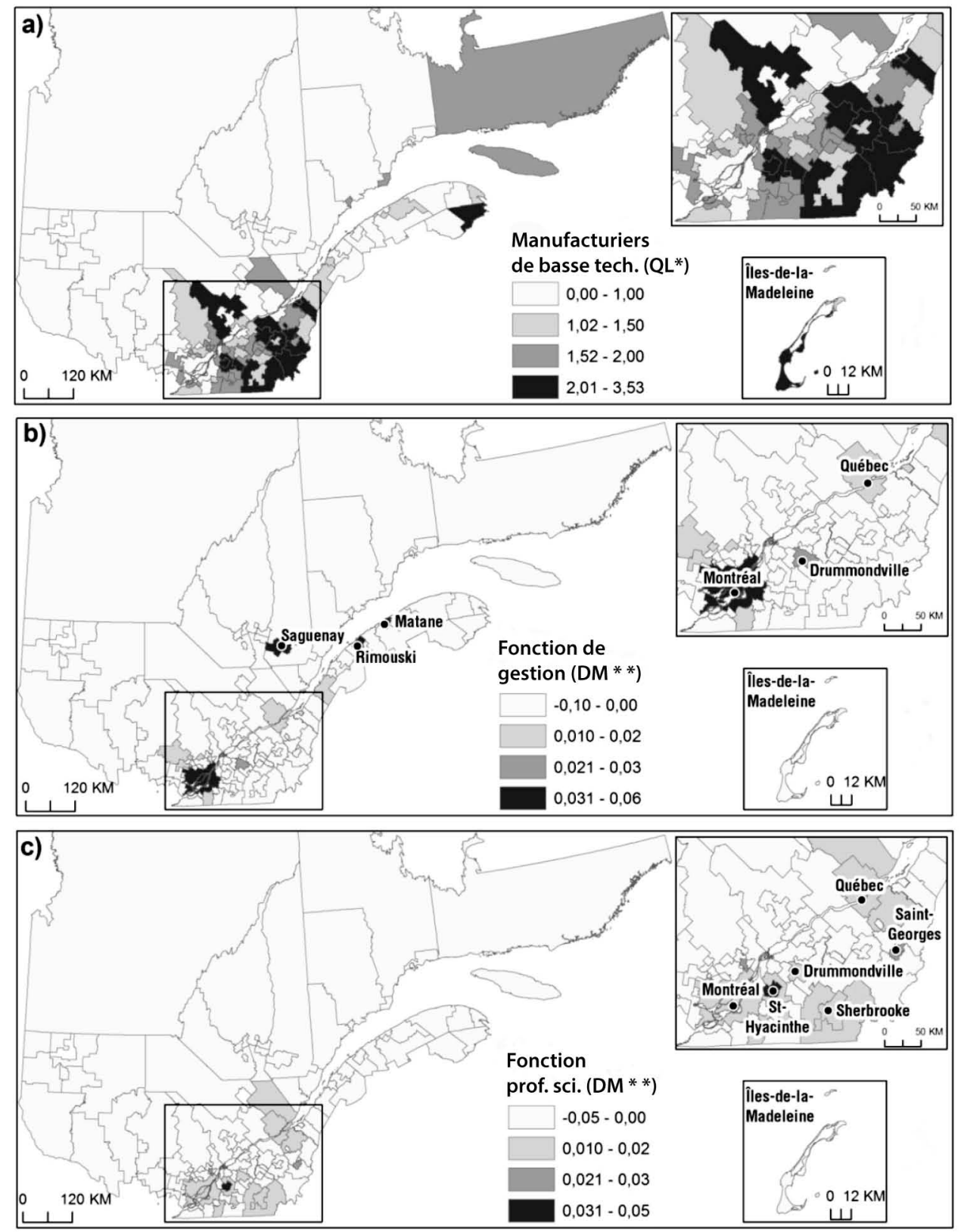

Source: Statistique Canada, Recensement de 2006; * $Q L$ - Quotient de localisation relatif à la moyenne canadienne; ${ }^{*}$ DD - Déviations de la moyenne québécoise. Cartographie Cédric Brunelle, 2012. 


\section{Un phénomène continu dans la hiérarchie urbaine}

\section{Le rôle de la taille urbaine et de la spécialisation sectorielle}

Malgré une importante concentration dans les régions métropolitaines, on constate que les fonctions à haut contenu en savoir affichent une localisation continue le long de la hiérarchie urbaine. Les figures 4 et 5 montrent, pour les données désagrégées, la relation entre les déviations de la moyenne pour les fonctions à haut contenu en savoir et, respectivement, la taille urbaine (en logarithme) et la spécialisation régionale dans le secteur de la production des biens (quotient de localisation). Un examen sommaire confirme que ces deux relations tiennent pour le système urbain québécois ${ }^{11}$. La figure 4 décrit une relation positive entre la concentration des fonctions à haut contenu en savoir et la taille urbaine ${ }^{12}$, alors que la figure 5 expose une relation négative avec la spécialisation régionale dans le secteur de la production des biens ${ }^{13}$. En continuité avec nos précédents résultats, ces relations nous permettent de dégager un premier constat.

Les figures montrent que la division spatiale entre les fonctions à haut contenu en savoir et de routine-production suit une distribution analogue à la structure spatiale retrouvée dans les industries de services, introduisant une dimension hiérarchique dans un secteur dont la géographie est a priori plus horizontale. Les résultats sont sur ce point univoques: les fonctions à haut contenu en savoir du secteur tendent à se localiser dans de plus grandes agglomérations et dans les régions où le secteur des services est dominant. Le phénomène corollaire est que les fonctions routinières et de production s'orientent vers les régions plus fortement spécialisées dans la production de biens. Comme nous le montre la figure 4, cette relation s'établit sous la forme d'un continuum, qui marque une ligne ascendante le long de la hiérarchie urbaine québécoise, partant de Matane, puis s'orientant vers Thetford-Mines, Victoriaville, Granby, Drummondville, Saguenay, Sherbrooke, Québec et Montréal. La relation inverse s'observe au niveau de la spécialisation dans le secteur de la production des biens. Les villes les moins spécialisées - donc davantage orientées vers les services concentrent une part plus importante de travailleurs à haut contenu en savoir à l'intérieur du secteur de la production des biens. La figure 5 révèle une droite descendante qui part des villes de services - Rimouski, Québec, Montréal, Rouyn-Noranda, passant par Saguenay, Trois-Rivières et Saint-Hyacinthe - pour s'orienter vers des centres fortement spécialisés dans la production des biens, dont Victoriaville, SorelTracy, La Tuque ou Baie-Comeau sont des exemples éloquents. En somme, les villes québécoises s'orientent dans un continuum où la taille urbaine et la spécialisation sectorielle semblent jouer un rôle sur la concentration des travailleurs à haut contenu en savoir dans le secteur de la production des biens.

11 Les mêmes tendances sont observées pour le système urbain canadien dans son ensemble.

12 Le coefficient de corrélation $(0,52)$ entre les deux variables est significatif au seuil de $99 \%$.

13 Le coefficient de corrélation $(-0,29)$ entre les deux variables est significatif au seuil de $95 \%$. 
Figure 4 Relation positive avec la taille urbaine - Spécialisation fonctionnelle dans le secteur de la production des biens*, agglomérations de recensement et régions métropolitaines de recensement, Québec, 2006

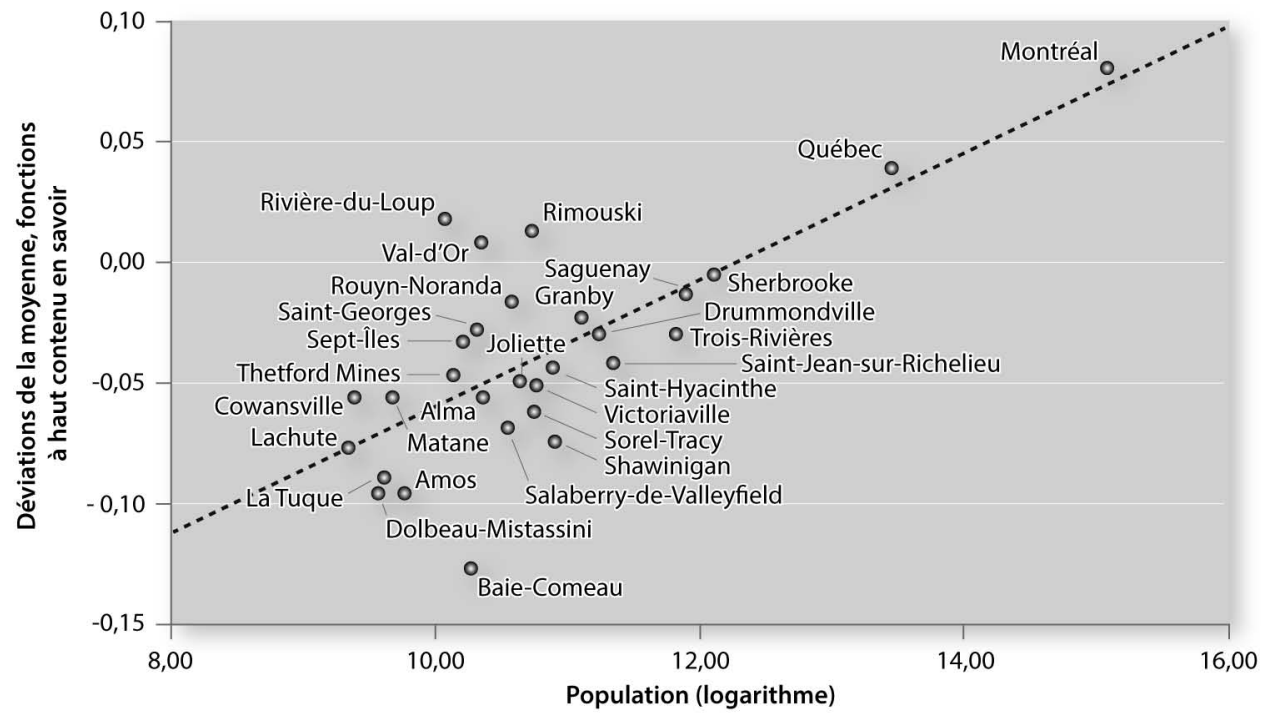

* Fonctions à haut contenu en savoir / fonctions routine et production - déviation de la moyenne

Figure 5 Relation négative avec la spécialisation dans la production des biens Spécialisation fonctionnelle dans le secteur de la production des biens*, agglomérations de recensement et région métropolitaines de recensement, Québec, 2006

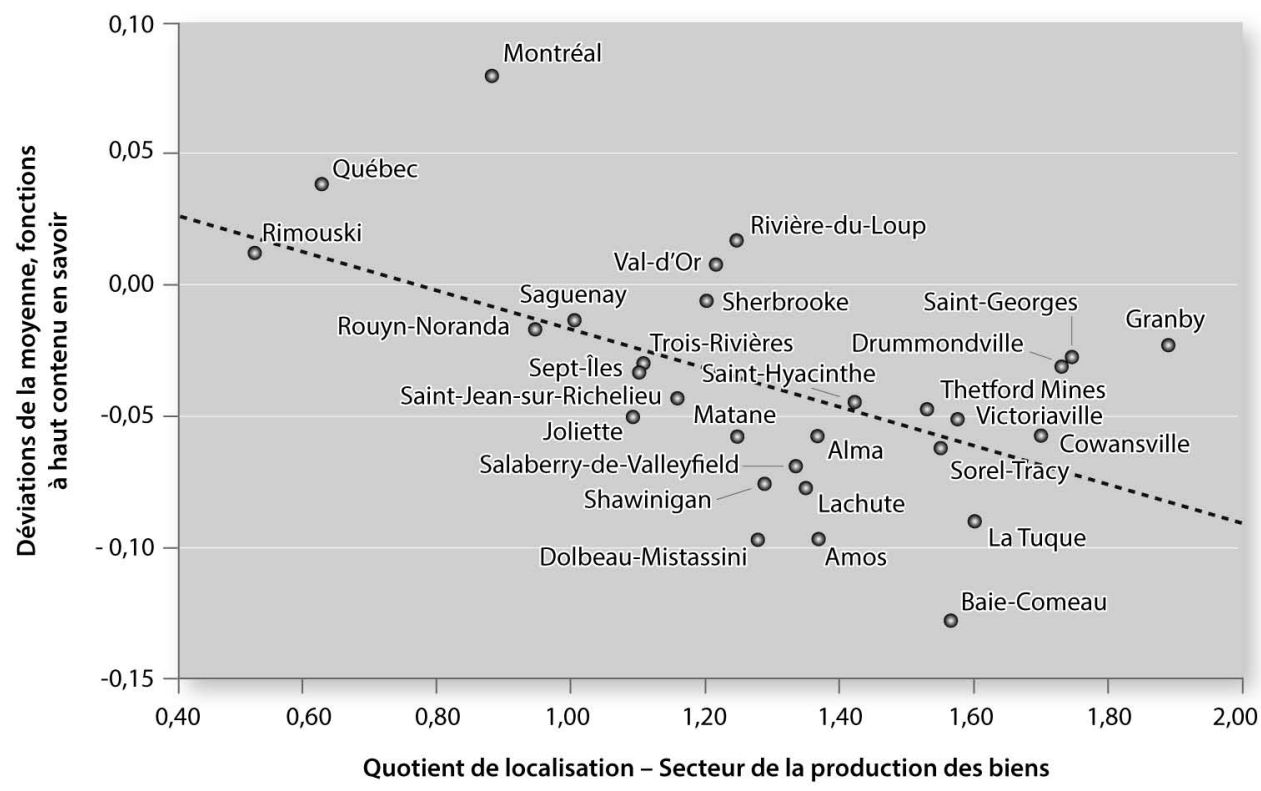

* Fonctions à haut contenu en savoir / fonctions routine et production - déviation de la moyenne 


\section{Des trajectoires régionales distinctes}

Un second constat s'impose. Pour de nombreuses villes, les écarts entre les valeurs observées aux figures 4 et 5 révèlent l'existence de distinctions importantes entre les régions. Certaines arborent des déviations positives relativement aux niveaux offerts par leur taille ou par leur degré de spécialisation, alors qu'en d'autres cas, on observe des niveaux qui leur sont systématiquement inférieurs. Baie-Comeau, La Tuque, Shawinigan, Amos, Dolbeau-Mistassini, Sorel-Tracy, Salaberry-de-Valleyfield, Lachute, Matane, Saguenay, Trois-Rivières et Saint-Jean montrent toutes des valeurs en dessous de la droite de corrélation. Ce résultat est corroboré par l'analyse de leur taux de croissance annuel moyen entre 1991 et 2006 (tableau 1). On y voit que, dans la plupart des cas, elles présentent des niveaux systématiquement inférieurs à la moyenne québécoise pour ce qui est du nombre de travailleurs attitrés à des fonctions à haut contenu en savoir. Cette trajectoire commune est le résultat de nombreux facteurs et nos résultats nous permettent d'explorer certaines pistes d'hypothèse. Il semble qu'une bonne part de ces villes ont - ou avaient ${ }^{14}$ - des structures économiques peu diversifiées. Ce fait n'est d'ailleurs pas étranger à la présence locale de grandes entreprises, dont AbitibiBowater (aujourd'hui Produits forestiers Résolu), Alcoa, Alcan (aujourd'hui Rio Tinto Alcan), Cascade Goodyear ou Gildan sont des exemples éloquents. De par leur taille, ces industries tendent à adopter des structures multiétablissements favorisant une fragmentation géographique de leurs activités, notamment à une échelle internationale. Plusieurs d'entre elles sont aussi confrontées à une concurrence féroce provenant des marchés extérieurs ${ }^{15}$. Non pas que la présence de ces grands employeurs entraîne une moins grande (dé)concentration locale de travailleurs à haut contenu en savoir, mais leur présence a, semble-t-il, favorisé une plus grande division spatiale de leurs fonctions. Polèse et al. (2002) et Polèse et Shearmur (2002 et 2005) ont déjà noté l'impact négatif des grandes entreprises sur le dynamisme économique régional, suscitant le syndrome du «rentier encombrant». Ce dernier décrit un effet de distorsion du marché local de l'emploi, qui se caractérise par des salaires élevés tendant à décourager la création ou l'implantation de PME manufacturières. Combiné à l'effet de la taille urbaine, la présence de grandes entreprises manufacturières pourrait avoir un effet répulsif analogue sur la concentration de travailleurs à plus haut contenu en savoir.

À l'opposé, plusieurs autres villes présentent des déviations positives systématiques. C'est notamment le cas de Rivière-du-Loup, Saint-Georges, Drummondville, Val-d’Or, Rimouski, Sept-Îles, Granby, Sherbrooke, Montréal et Québec, où la production des biens concentre une part importante de travailleurs à haut contenu en savoir. Hormis Val-d'Or, ces villes possèdent des niveaux de croissance qui, dans l’ensemble, sont supérieurs à la médiane québécoise sur la période (tableau 1).

14 Trois-Rivières et Saint-Jean-sur-Richelieu sont deux exceptions, s'étant, pour des raisons différentes, profondément et rapidement transformées au cours des 10 dernières années - la première sortant de l'industrie des pâtes et papiers et la seconde s'orientant dans une économie de services de plus en plus intégrée avec la RMR de Montréal.

15 Les fermetures de Gildan et de Goodyear en 2006 et 2007 à Salaberry-de-Valleyfield en sont de bons exemples. Nos données étant pour 2006, ces employeurs étaient intégrés dans les tendances relatées dans les figures. 
Tableau 1 Évolution des effectifs, fonctions à haut contenu en savoir - secteur de la production des biens. Agglomérations de recensement, Québec, 1991-2006

\begin{tabular}{|c|c|c|c|c|c|}
\hline Agglomération & 1991 & 1996 & 2001 & 2006 & $\begin{array}{l}\text { Taux de croissance } \\
\text { relatif moyen }\end{array}$ \\
\hline Rivière-du-Loup & 139 & 212 & 495 & 540 & $9,5 \%$ \\
\hline Saint-Georges & 223 & 405 & 670 & 855 & $9,4 \%$ \\
\hline Drummondville & 619 & 925 & 1485 & 1910 & $7,8 \%$ \\
\hline Victoriaville & 383 & 568 & 630 & 950 & $6,2 \%$ \\
\hline Saint-Hyacinthe & 504 & 575 & 690 & 1070 & $5,1 \%$ \\
\hline Joliette & 260 & 417 & 410 & 540 & $5,0 \%$ \\
\hline Sherbrooke & 1678 & 2308 & 3155 & 3450 & $4,9 \%$ \\
\hline Québec & 4884 & 6083 & 7755 & 9425 & $4,5 \%$ \\
\hline Thetford Mines & 248 & 373 & 460 & 465 & $4,3 \%$ \\
\hline Alma & 286 & 273 & 340 & 485 & $3,6 \%$ \\
\hline Matane & 131 & 126 & 150 & 215 & $3,4 \%$ \\
\hline Sept-Îles & 256 & 282 & 480 & 405 & $3,1 \%$ \\
\hline Rimouski & 261 & 324 & 305 & 410 & $3,1 \%$ \\
\hline Montréal & 48131 & 54697 & 70255 & 75000 & $3,0 \%$ \\
\hline Granby & 1431 & 1506 & 2105 & 2030 & $2,4 \%$ \\
\hline Trois-Rivières & 1516 & 1752 & 1915 & 2040 & $2,0 \%$ \\
\hline Lachute & 115 & 108 & 110 & 150 & $2,0 \%$ \\
\hline Shawinigan & 477 & 624 & 740 & 620 & $1,8 \%$ \\
\hline Val-d'Or & 505 & 578 & 620 & 655 & $1,7 \%$ \\
\hline Dolbeau-Mistassini & 108 & 185 & 240 & 135 & $1,5 \%$ \\
\hline Saint-Jean-sur-Richelieu & 1132 & 1019 & 1075 & 1395 & $1,4 \%$ \\
\hline Salaberry-de-Valleyfield & 452 & 395 & 525 & 535 & $1,1 \%$ \\
\hline Amos & 258 & 232 & 330 & 275 & $0,4 \%$ \\
\hline Cowansville & 241 & 175 & 300 & 250 & $0,3 \%$ \\
\hline Saguenay & 2133 & 1797 & 2080 & 2155 & $0,1 \%$ \\
\hline Sorel-Tracy & 860 & 908 & 940 & 785 & $-0,6 \%$ \\
\hline Rouyn-Noranda & 651 & 504 & 645 & 535 & $-1,3 \%$ \\
\hline Baie-Comeau & 428 & 498 & 425 & 300 & $-2,3 \%$ \\
\hline La Tuque & 273 & 256 & 260 & 180 & $-2,7 \%$ \\
\hline Total & 68583 & 78103 & 99590 & 107760 & $3,1 \%$ \\
\hline
\end{tabular}

Source: Statistique Canada, recensements de 1971-2006 - compilation spéciale LASER

Ces résultats ne sont pas en soi nouveaux pour les RMR de Montréal, Québec et Sherbrooke. On remarque en revanche des résultats étonnants dans d'autres régions de prime abord moins bien positionnées. Ainsi, les plus fortes croissances entre 1991 et 2006 se produisent en dehors des grandes agglomérations métropolitaines du Québec, dans les villes moyennes de Rivière-du-Loup, Saint-Georges, Drummondville, Victoriaville, Saint-Hyacinthe, Thetford Mines et Joliette. Sauf cette dernière, on constate qu'elles partagent une localisation dans l'axe du sud-est québécois - région caractérisée par un dynamisme industriel unique à l'échelle nord-américaine (Lemelin, 2008). L’un des aspects-clés de la croissance observée dans ces villes tient à la densité de 
leur tissu industriel, composé en grande partie de petites et moyennes entreprises (PME) locales. Si le cas de Saint-Georges-de-Beauce reste l'un des mieux documentés (Doloreux et al., 2005; Julien, 1997 et 2005), une littérature émergente indique des dynamiques analogues dans plusieurs autres régions de la province. Drummondville, Victoriaville, Granby et Saint-Hyacinthe ont plus récemment été associées à une forte croissance économique (Polèse, 2009; Proulx, 2009). Nos résultats contribuent à soutenir ces tendances alors qu'ils montrent que la production des biens y subit une transition accélérée vers les fonctions à plus haut contenu en savoir. La principale hypothèse retenue, soulignée dans de nombreux travaux, est que cette dynamique est en grande partie alimentée par l'effet positif de l'entrepreneuriat local sur le processus de développement économique régional (Doloreux et al., 2005 ; Apparicio et al., 2009). Ce constat marque une distinction importante entre la PME et la grande entreprise, à savoir que l'entrepreneur local est presque par définition attitré aux fonctions stratégiques de son entreprise. Au contraire, la grande corporation ne garantit jamais l'enracinement local des fonctions décisionnelles - les logiques de dépendance régionale qui peuvent en résulter ayant déjà fait l'objet de fortes critiques (Aydalot, 1976; Bade, 1983 ; Sallez, 1983 ; Massey, 1984). Par contre, ces dynamiques n’expliquent pas à elles seules la concentration des fonctions à haut contenu en savoir. Sept-Îles offre un bon contre-exemple. Loin d'une synergie entrepreneuriale, la croissance y semble davantage attribuable à l'effet combiné de la hausse du prix des matières premières et d'investissements massifs dans des infrastructures stratégiques, dont plusieurs sont le fruit de grandes entreprises ${ }^{16}$.

D’autres cas recèlent leur part de mystère. Celui de Québec étant à tout le moins résolu - Polèse (2012) attribue l'essor économique de Québec à la «dé-fonctionnarisation» de son marché du travail -, ceux de Rivière-du-Loup et dans une moindre mesure de Rimouski restent à documenter. Malgré leur éloignement relatif, ces deux villes affichent des niveaux de croissance étonnants sur la période. Alors que l'entrepreneuriat paraît aussi y jouer un rôle, l'hypothèse plus particulière d'une "progression" des entreprises locales le long de leur "chaîne de valeur» (OCDE, 2007; Sturgeon et Gereffi, 2009) mériterait d'être approfondie - la filière de la tourbe offrant un exemple frappant d'une telle dynamique ${ }^{17}$. Une seconde piste pointe vers les facteurs institutionnels et politiques qui ont pu favoriser le développement d'entreprises locales à plus forte valeur ajoutée au Bas-Saint-Laurent, notamment dans le créneau maritime (Doloreux et al., 2007 ; Doloreux et Melançon, 2008).

En somme, nos résultats soulignent des trajectoires régionales distinctes. Malgré une baisse relative des effectifs dans certaines régions, plusieurs autres affichent des niveaux de croissance systématiquement supérieurs aux agglomérations métropolitaines. S'ils sont généralisés, ces résultats tendent à nuancer les résultats précédents où la déconcentration manufacturière des 20 dernières années semblait s'être en partie limitée aux fonctions routinières et de production (figure 1). Les analyses désagrégées nous poussent à considérer qu'un phénomène de rattrapage soit à l'œuvre, suggérant une convergence régionale des fonctions à moyen terme.

16 L’aluminerie Alouette de Sept-Îles en offre un exemple éloquent.

17 Dans ce créneau, l'entreprise Premier Tech de Rivière-du-Loup représente sans nul doute le meilleur exemple de mobilité dans la chaîne de valeur. Partant d'une activité d'extraction de la mousse de sphaigne, l'entreprise a non seulement innové en diversifiant ses produits dérivés (horticulture, emballage, technologies de traitement des eaux, etc.), mais a aussi œuvré au développement, à la fabrication locale et à l'exportation internationale de machinerie spécialisée. 


\section{Vers une convergence régionale?}

Cette section teste l'hypothèse de la convergence régionale des fonctions à haut contenu en savoir à l'intérieur du secteur de la production des biens, de 1971 à 2006. Cinq séries de régressions linéaires y sont réalisées. Les résultats sont présentés au tableau 2. Les données sont organisées dans un panel de 145 observations, couvrant cinq périodes temporelles de 1981 à $2006^{18}$. Un modèle en panel a été choisi afin d'allouer un nombre suffisant de degrés de liberté pour l'introduction de variables de contrôle dans les estimations.

À des fins de comparabilité longitudinale, notre échantillon se limite aux 29 agglomérations présentées au tableau 1. Ce choix implique un biais: plusieurs petites municipalités et communautés rurales sont exclues de l'analyse. Les résultats ne peuvent donc être généralisés au système urbain québécois dans son ensemble. Malgré tout, le test s'avère être un puissant complément aux précédentes analyses. Il permet d'évaluer la convergence dans les régions du Québec à prédominance urbaine, soit là où le phénomène paraît le plus probable.

La variable dépendante retenue est le taux de croissance annuel moyen (TCAM $\left.{ }_{j, t-t-1}\right)$ des effectifs pour les fonctions à haut contenu en savoir entre la période courante $t$ et la période initiale $t-1$ (équation [004], annexe 5) ${ }^{19}$. Notre hypothèse est plus directement testée par la variable Prop $_{i t-1}$, qui représente la part des effectifs régionaux dans les fonctions à haut contenu en savoir sur l'emploi total du secteur de la production des biens à la période initiale $t$-1. La convergence régionale implique des coefficients négatifs: l'hypothèse consistant à affirmer que la croissance est moins importante dans les régions initialement dotées d'une part supérieure d'emploi dans ces fonctions.

Notre hypothèse est soumise à l'introduction de variables de contrôle. Suivant les résultats des figures 4 et 5 , nous retenons la taille urbaine (en logarithme) et le niveau de spécialisation sectorielle dans la production des biens (quotient de localisation). La structure en panel permet l'introduction de variables dichotomiques et d'interaction pour chaque période temporelle. Les variables dichotomiques servent à capter les changements structurels - conjoncture économique, changement technologique, législations provinciales - qui pourraient faire varier le taux de croissance dans le temps pour l'ensemble des agglomérations. De leur côté, les variables d'interaction permettent d'observer si l'effet de notre variable de test augmente ou diminue relativement à l'année de référence, ici fixée à 1981.

Pleinement spécifié, le modèle intègre des effets fixes (EF) pour chaque agglomération, ce qui requiert davantage d'explications. Chaque ville est distinctement caractérisée par un ensemble de facteurs idiosyncrasiques - infrastructures locales, position géographique, structure démographique, politiques locales - souvent difficilement mesurables. Ces facteurs n'étant pas spécifiés dans notre modèle, leur omission n’est pas sans conséquence: elle aurait pour effet de biaiser nos estimations dans le cas où

18 L'année 1971 est omise afin de calculer les taux de croissance et les valeurs initiales des variables indépendantes sur la période.

19 Les modèles n’ont pas été ventilés pour chaque fonction (gestion; professionnels/scientifiques). Ce choix a dû être fait compte tenu du nombre élevé de cellules étant dans une zone critique du point de vue de l'erreur type en début de période (1971), lorsqu'une tripartition est réalisée entre la ville, la fonction et l'industrie. Des analyses désagrégées ont montré des relations similaires à celles présentées au tableau 2. 
ils seraient simultanément corrélés avec le taux de croissance et la variable de test. Cette situation semble hautement probable. Comme nous ne pouvons directement mesurer ces facteurs, mais désirons contrôler leur effet sur la variable de test, une stratégie répandue consiste à introduire des effets fixes (EF) pour chaque observation. Sans pouvoir être directement interprétés, ces effets permettent d'évacuer du modèle l'ensemble des facteurs qui varient entre lieux, mais restent constants dans le temps (Wooldridge, 2002; Greene, 2005 ; Stock et Watson, 2007). Réalisés ultérieurement, des tests statistiques ont révélé la présence de tels effets et confirmé ce choix relativement à un modèle avec effets aléatoires ${ }^{20}$. En dernière instance, le test du I de Moran (distance inverse carrée) n’a pas révélé la présence d’autocorrélation spatiale dans nos modèles, ce qui reflète la distance géographique relativement élevée qui sépare les villes de notre échantillon. Les tests d'hétéroscédasticité et de multicolinéarité ont aussi été réalisés et les correctifs mis en place lorsque nécessaires ${ }^{21}$. À sa pleine extension, le modèle prend la forme suivante:

TCAM $_{j, t-t-1}=\beta_{0}+\beta_{1 t} \operatorname{Prop}_{j t-1}+\beta_{2 t} L n P O P_{j t-1}+\beta_{3 t} L Q_{t-1}+\gamma_{t}+\alpha_{j}+u_{\mathrm{jt}} \quad$ [001]

Où

$T C A M_{j, t-t-1}=$ Pour chaque ville $j$, taux de croissance annuel moyen des effectifs pour les fonctions à haut contenu en savoir entre la période courante $t$ et la période initiale $t-1$. Secteur de la production des biens, 1971-2006. Équation [004] de l'annexe 5.
Prop $_{j t-1}$
$=$ Pour chaque ville $j$, part des effectifs dans les fonctions à haut contenu en savoir sur l'emploi total du secteur de la production des biens à la période initiale $t-1$.
$L n P O P_{j t-1}=$ Pour chaque ville $j$, taille de la population à la période initiale $t-1$, en logarithme.
$L Q_{j t-1} \quad=$ Pour chaque ville $j$, quotient de localisation du secteur de la production des biens à la période initiale $t-1$. Équation [003] de l'annexe 5.
$\gamma_{t} \quad=$ Variables dichotomiques pour chaque période (effets fixes de temps) de 1981 à 2006 (référence fixée à 1981)
$\beta_{\text {Prop } \_t} \quad=$ Vecteur de coefficients à être estimé pour la variable Prop Prt-. $_{1}$. Varie pour chaque période $t$ (variable d'interaction - référence fixée à 1981).
$\alpha_{j} \quad=$ Effets fixes de villes.
$u_{\mathrm{jt}} \quad=$ Les termes d'erreur supposés indépendants et identiquement distribués.

20 Pour l'ensemble des modèles dans un intervalle de confiance à 95\%, un test de probabilité jointe a révélé que des effets fixes de temps étaient nécessaires. Le test de Hausman a été significatif, suggérant l'utilisation d'un modèle avec effets fixes; le test de Breusch-Pagan n'était pas significatif au seuil de 0,05 .

21 La présence d'hétéroscédasticité et de corrélations temporelles nous a fait opter pour l'utilisation d'erreurs standards robustes de type Newey-West. Les diagnostics de multicolinéarité (FIV; Tolérance) n’ont pas révélé de valeurs au-dessus de cinq pour les modèles sans effets fixes. 
Le tableau 2 montre les coefficients standardisés pour l'ensemble des modèles. Le modèle 1 n'inclut que la variable de test $\left(\operatorname{Prop}_{j t-1}\right)$ alors que le modèle 2 augmente ce dernier par l'ajout des variables de taille urbaine $\left(\operatorname{LnPOP}_{j t-1}\right)$ et de spécialisation sectorielle $\left(L Q_{j t-1}\right)$. Afin de contrôler les effets temporels, le modèle 3 intègre des variables dichotomiques pour chaque période, avec l’année 1981 en référence. Le modèle 4 inclut les effets fixes (EF) pour chaque ville moins une, afin d'éviter la multicolinéarité parfaite. Le modèle 5 ajoute des variables d'interaction à chaque période pour la variable de test.

Tableau 2 Test de convergence: croissance annuelle moyenne sur proportions initiales en début de période - Fonctions à haut contenu en savoir. Secteur de la production des biens, Québec, 1971-2006

\begin{tabular}{|c|c|c|c|c|c|}
\hline & \multicolumn{5}{|c|}{ Haut contenu en savoir } \\
\hline & Modèle 1 & Modèle 2 & Modèle 3 & Modèle 4 & Modèle 5 \\
\hline Prop $_{t-1}$ & $\begin{array}{r}-0,37 \\
(0,000)^{* * *}\end{array}$ & $\begin{array}{r}-0,45 \\
(0,000)^{* * *}\end{array}$ & $\begin{array}{r}-0,765 \\
(0,000)^{* * *}\end{array}$ & $\begin{array}{r}-1,45 \\
(0,000) * * *\end{array}$ & $\begin{array}{r}-2,132 \\
(0,000)^{* * *}\end{array}$ \\
\hline $\operatorname{LnPOP}_{t-1}$ & $\cdots$ & $\begin{array}{r}0,11 \\
(0,163)\end{array}$ & $\begin{array}{r}0,201 \\
(0,006) * * *\end{array}$ & $\begin{array}{r}2,59 \\
(0,002) * * *\end{array}$ & $\begin{array}{r}2,416 \\
(0,005)^{* * *}\end{array}$ \\
\hline$Q_{\mathrm{jt}-1}$ & $\cdots$ & $\begin{array}{r}-0,19 \\
(0,021)^{* *}\end{array}$ & $\begin{array}{r}-0,246 \\
(0,002)^{* * *}\end{array}$ & $\begin{array}{r}-0,05 \\
(0,847)\end{array}$ & $\begin{array}{r}0,163 \\
(0,475)\end{array}$ \\
\hline \multicolumn{6}{|c|}{ Dichotomique de temps (référence = 1981) } \\
\hline 1991 & $\cdots$ & $\cdots$ & $\begin{array}{r}0,09 \\
(0,218)\end{array}$ & $\begin{array}{r}0,13 \\
(0,118)\end{array}$ & $\begin{array}{r}-0,101 \\
(0,678)\end{array}$ \\
\hline 1996 & $\cdots$ & $\cdots$ & $\begin{array}{r}0,427 \\
(0,000) * * *\end{array}$ & $\begin{array}{r}0,60 \\
(0,000)^{* * *}\end{array}$ & $\begin{array}{r}0,734 \\
(0,036) * *\end{array}$ \\
\hline 2001 & $\cdots$ & $\cdots$ & $\begin{array}{r}0,32 \\
(0,006)^{* * *}\end{array}$ & $\begin{array}{r}0,62 \\
(0,000)^{* * *}\end{array}$ & $\begin{array}{r}0,074 \\
(0,799)\end{array}$ \\
\hline 2006 & $\cdots$ & $\ldots$ & $\begin{array}{r}0,43 \\
(0,001)^{* * *}\end{array}$ & $\begin{array}{r}0,79 \\
(0,000)^{* * *}\end{array}$ & $\begin{array}{r}0,154 \\
(0,593)\end{array}$ \\
\hline \multicolumn{6}{|c|}{ Interaction (référence = 1981) } \\
\hline $\operatorname{Prop}_{\mathrm{t}-1}{ }^{*} 1991$ & $\cdots$ & $\cdots$ & $\cdots$ & $\cdots$ & $\begin{array}{r}0,373 \\
(0,183)\end{array}$ \\
\hline $\operatorname{Prop}_{\mathrm{t}-1}{ }^{*} 1996$ & $\cdots$ & $\cdots$ & $\cdots$ & $\cdots$ & $\begin{array}{r}0,167 \\
(0,686)\end{array}$ \\
\hline $\operatorname{Prop}_{\mathrm{t}-1} * 2001$ & $\cdots$ & $\cdots$ & $\cdots$ & $\cdots$ & $\begin{array}{r}1,016 \\
(0,029) * *\end{array}$ \\
\hline $\operatorname{Prop}_{\mathrm{t}-1} * 2006$ & $\cdots$ & $\cdots$ & $\cdots$ & $\cdots$ & $\begin{array}{r}1,187 \\
(0,020)^{* *}\end{array}$ \\
\hline Effets fixes (EF) & Non & Non & Non & Oui & Oui \\
\hline $\mathbf{N}$ & 145 & 145 & 145 & 145 & 145 \\
\hline Prob $>F$ & $(0,000) * * *$ & $(0,000)^{* * *}$ & $(0,000)^{* * *}$ & $(0,000)^{* * *}$ & $(0,000)^{* * *}$ \\
\hline $\mathbf{R}^{2}$ & 0,14 & 0,19 & 0,31 & 0,51 & 0,57 \\
\hline
\end{tabular}

***, ${ }^{* *}$ et * pour valeurs significatives aux seuils de confiance de $1 \%, 5 \%$ et $10 \%$. Coefficients standardisés. Erreurs standards robustes à l'hétéroscédasticité et à l'autocorrélation temporelle (Newey-West). 
A priori, les résultats des régressions suggèrent qu'entre 1971 et 2006, les fonctions à haut contenu en savoir tendent à converger entre les agglomérations du Québec. Les coefficients pour la part initiale des effectifs dans les fonctions à haut contenu en savoir $\left(\right.$ Prop $\left._{j t-1}\right)$ montrent une relation négative et significative pour tous les modèles, ce qui semble valider l'hypothèse de convergence. On doit néanmoins prendre garde de tirer une conclusion trop hâtive. Bien que cette relation soit croissante avec l'ajout de variables de contrôle dans les modèles 2 et 3 - les variables de taille urbaine et les dichotomiques d'années captant une part de l'effet positif contenu dans la variable test -, l'introduction d'effets fixes (EF) révèle que la convergence reste inférieure à l'effet positif de la taille urbaine sur les taux de croissance ${ }^{22}$.

Cette dynamique tend par ailleurs à s'accentuer dans le temps. L'ajout d'interactions temporelles sur la variable Prop $_{j t-1}$ montre que son effet négatif sur les taux de croissance diminue sur la période: les coefficients sont positifs et significatifs à partir de 2001 relativement à l'année de référence. Ces résultats laissent présager que l'arrivée des technologies de communication et l'ouverture des marchés ont pu freiner l'attraction et la rétention de fonctions à plus haut contenu en savoir dans les plus petites agglomérations de la province. Sans supprimer l'effet dominant de la taille urbaine, on note donc un certain niveau de convergence entre 1971 et 1996, suivi d'une période de concentration dans les grandes agglomérations urbaines entre 1996 et 2006. Cela illustre l'effet croissant des forces centripètes sur les activités à plus fort niveau de capital humain, donnant crédit aux travaux qui ont relevé l'importance des économies d’agglomération dans ce processus (Scott, 2010; Storper, 2010).

On observe aussi que la spécialisation dans le secteur de la production des biens $\left(Q L_{i t-1}\right)$ a un effet négatif sur les niveaux de croissance dans les modèles 2 et 3 , mais qu'elle n'est plus significative après l'introduction des EF. D'un simple point de vue technique, cet ajustement souligne l'existence de facteurs non observés qui sont à la fois corrélés avec la spécialisation sectorielle et les taux de croissance. Plus directement, le modèle indique que l'effet négatif de la spécialisation dans le secteur de la production des biens est capté par d'autres variables. Alors que l'intégration d'EF dans le modèle reste nécessaire pour estimer adéquatement les coefficients du modèle, les implications théoriques qui en découlent imposent leur part de réflexion. La plus fondamentale touche à la structure sectorielle des économies régionales. Les résultats montrent que la concentration des fonctions à haut contenu en savoir s'effectue indépendamment des activités du secteur, relativement à d'autres facteurs. Parmi plusieurs de ces facteurs, la littérature pointe vers le niveau régional de capital humain, dont l'effet reste ici difficile à estimer en vertu de son endogénéité relativement aux taux de croissance observés. Hendricks (2011) montre que les villes hautement qualifiées emploient un nombre important d'employés qualifiés dans toutes les industries, $80 \%$ des variations entre les villes américaines étant expliquées par des différences à l'intérieur des industries. La structure industrielle y a donc moins à jouer que l'effet combiné d'une concentration locale de capital humain, sinon que d'une diversité d'activité favorisant des économies externes aux secteurs, mais internes aux catégories de connaissances, ce que d'autres ont qualifié d'économies de «juxtaposition» (Shearmur et Polèse, 2005) ou

22 Tel qu'envisagé, ces résultats témoignent de l'existence de nombreux autres facteurs n'étant pas directement mesurés dans les modèles, mais dont l'omission aurait pour effet de biaiser les estimations. L'analyse du $\mathrm{R}^{2}$ offre une mesure de leur impact, ce dernier passant de 0,19 à 0,51 , suivant l'intégration des variables dichotomiques temporelles et des EF. 
de «variétés reliées» (Boschma et Iammarino, 2009). D’une certaine façon, on peut présumer que, dans les modèles proposés, le capital humain est indirectement pris en compte par la variable de taille urbaine, ce qui explique en partie qu'elle y joue le principal rôle, notamment face à la spécialisation dans la production des biens.

En résumé, la convergence régionale des fonctions à haut contenu en savoir ne permet pas de renverser l'effet croissant de la taille urbaine dans les agglomérations du Québec. Si nos résultats montrent qu'un certain niveau de convergence a lieu entre 1971 et 1996, on observe que cet effet reste inférieur à celui de la taille urbaine et s'estompe à partir de 1996. Ces résultats nous paraissent cohérents avec les précédents (figure 1) et avec les tendances rapportées dans la littérature. En dernier lieu, la spécialisation sectorielle ne semble pas avoir d'impact lorsqu'on intègre les EF, ce qui laisse présager l'existence d'autres facteurs explicatifs.

\section{Conclusion}

Cet article avait pour objectif d'identifier les tendances dans la concentration des activités à haut contenu en savoir au sein du secteur de la production des biens au Québec et d'évaluer l'impact potentiel de ces dynamiques sur les trajectoires de développement économique régional. La littérature suggère que la déconcentration des activités industrielles hors des grandes agglomérations urbaines du Québec a pu être alimentée par la fragmentation fonctionnelle des activités du secteur. Le résultat envisagé est un renforcement des agglomérations métropolitaines de la province dans les segments à plus forte intensité en savoir, qui sous-tend une divergence économique croissante des régions du Québec.

Notre étude montre que les fonctions à haut contenu en savoir restent très fortement concentrées à Montréal et à Québec. Les tendances s'orientent vers une accélération du phénomène après 1991, et ce, dans la plupart des sous-secteurs. Contrairement à une déconcentration généralisée de l'industrie manufacturière, ces résultats appuient donc l'idée selon laquelle la décentralisation de la production des biens a pu en grande partie se limiter aux fonctions de routine et de production.

De façon désagrégée, plusieurs réalités semblent toutefois nuancer cette analyse. Les taux de croissance élevés observés dans les villes de l'arc industriel et la plus forte concentration dans certaines agglomérations périphériques révèlent l'existence de trajectoires distinctes dans de nombreuses régions du Québec. La causalité qui leur est sous-jacente est sans doute complexe et nos analyses ne nous permettent que d'émettre certaines hypothèses. La plus probante est liée aux caractéristiques intrinsèques de l'arc industriel québécois. Une piste d'analyse tient à l'effet positif des PME locales qui, conformément à une capacité de fragmentation géographique plus restreinte, favorisent une colocalisation régionale des fonctions à haut contenu en savoir. Cette hypothèse est par ailleurs renforcée par les faibles taux de croissance observés dans les régions où la grande entreprise est dominante. D'autres cas de figure s'y rattachent. Certains pointent vers la capacité des entreprises locales à "progresser» dans la chaîne de valeur. D’autres soulèvent le rôle que jouent les facteurs géographiques, institutionnels et politiques dans le processus de spécialisation fonctionnelle. Force est de constater que les éléments idiosyncrasiques y restent nombreux. Leur intérêt est certes manifeste pour l'élaboration de diagnostics et de stratégies régionales. 
Malgré tout, le spectre d'une asymétrie économique croissante entre les régions du Québec reste bien réel. Nos résultats indiquent que la bonne performance affichée dans plusieurs villes ne suffit pas à renverser la concentration métropolitaine des fonctions à plus haut contenu en savoir. Les tests sont sur ce point univoques: l'effet de convergence reste inférieur à celui de la taille urbaine dans les agglomérations du Québec et tend à diminuer avec le temps. Les conséquences sont nombreuses. Outre le renforcement stratégique des villes où se concentrent les fonctions à haut contenu en savoir, ces résultats laissent présager une division spatiale grandissante entre les niveaux régionaux de capital humain et de productivité. Sur le marché du travail, les effets potentiels pointent vers de nouvelles inégalités interrégionales touchant les revenus et, de façon indirecte, la croissance locale de l'emploi - le multiplicateur régional pour les biens et services étant plus important dans les régions où les revenus sont élevés. Par leur aspect cumulatif, ces processus sont à même d'exacerber la vulnérabilité des entreprises et des régions dont les conditions permettent plus difficilement d'attirer et de retenir les segments productifs à plus forte croissance.

En dernière analyse, les résultats présentés dans cet article révèlent une transformation majeure dans l'organisation géographique de l'activité industrielle. Les régions passent d'une logique territoriale axée sur la production de biens finaux à une spécialisation économique reposant sur les segments intermédiaires le long de la chaîne verticale de production. Cette transition soulève en outre de nombreuses questions n'étant pas directement traitées dans cet article. Des analyses plus fines seraient nécessaires pour formaliser les facteurs permettant de développer et de soutenir la présence de fonctions à plus haut contenu en savoir dans les régions non métropolitaines. D’autres études pourraient se pencher sur l'impact de cette transformation sur la génération de nouvelles disparités à l'échelle régionale. La compréhension de ces dynamiques semble nécessaire pour l'élaboration de politiques publiques touchant le développement des entreprises et des régions.

\section{Remerciements}

Je tiens à remercier Stéphane Pronovost et Luc Vallée de Développement Économique Canada, qui ont grandement enrichi cette recherche par leurs commentaires et suggestions. Merci à Wilfried Koch de l'UQAM pour ses conseils utiles lors des séminaires LASER. Mes remerciements vont également à Mario Polèse pour ses commentaires sur la première version de ce texte, ainsi qu'aux trois évaluateurs anonymes dont les conseils ont grandement enrichi cette recherche. Toute erreur restante est entièrement mienne. 
Annexe 1 Emploi - total, secteur production des biens et fonctions à haut contenu en savoir dans le secteur. Évolution dans le système urbain québécois, 1971-2006

\begin{tabular}{|c|c|c|c|c|c|c|c|c|c|}
\hline Emploi & $\begin{array}{l}\text { Villes / } \\
\text { Régions }\end{array}$ & 1971 & 1981 & 1991 & 1996 & 2001 & 2006 & $\begin{array}{l}\text { Taux } \\
\text { croiss. } \\
\text { annuel } \\
\text { moyen } \\
71-91\end{array}$ & $\begin{array}{l}\text { Taux } \\
\text { croiss. } \\
\text { annuel } \\
\text { moyen } \\
91-06\end{array}$ \\
\hline \multirow[t]{8}{*}{ Total } & Prov. QC & 1843900 & 2549975 & 2930190 & 2968040 & 3338270 & 3572380 & $2,3 \%$ & $1,3 \%$ \\
\hline & Montréal & $\begin{array}{r}955308 \\
51,8 \%\end{array}$ & $\begin{array}{r}1282979 \\
50,3 \%\end{array}$ & $\begin{array}{r}1457636 \\
49,7 \%\end{array}$ & $\begin{array}{r}1460725 \\
49,2 \%\end{array}$ & $\begin{array}{r}1661145 \\
49,8 \%\end{array}$ & $\begin{array}{r}1827060 \\
51,1 \%\end{array}$ & $2,1 \%$ & $1,5 \%$ \\
\hline & Québec & $\begin{array}{r}166304 \\
9,0 \%\end{array}$ & $\begin{array}{r}246539 \\
9,7 \%\end{array}$ & $\begin{array}{r}312248 \\
10,7 \%\end{array}$ & $\begin{array}{r}314535 \\
10,6 \%\end{array}$ & $\begin{array}{r}345770 \\
10,4 \%\end{array}$ & $\begin{array}{r}382235 \\
10,7 \%\end{array}$ & $3,2 \%$ & $1,3 \%$ \\
\hline & Sherb. & $\begin{array}{r}56756 \\
3,1 \%\end{array}$ & $\begin{array}{r}82661 \\
3,2 \%\end{array}$ & $\begin{array}{r}97192 \\
3,3 \%\end{array}$ & $\begin{array}{r}101096 \\
3,4 \%\end{array}$ & $\begin{array}{r}118705 \\
3,6 \%\end{array}$ & $\begin{array}{r}122670 \\
3,4 \%\end{array}$ & $2,7 \%$ & $1,3 \%$ \\
\hline & Tr.-Riv. & $\begin{array}{r}51413 \\
3 \%\end{array}$ & $\begin{array}{r}68265 \\
3 \%\end{array}$ & $\begin{array}{r}80127 \\
3 \%\end{array}$ & $\begin{array}{r}79831 \\
3 \%\end{array}$ & $\begin{array}{r}85715 \\
3 \%\end{array}$ & $\begin{array}{r}89280 \\
2 \%\end{array}$ & $2,2 \%$ & $0,7 \%$ \\
\hline & Saguenay & $\begin{array}{r}71907 \\
3,9 \%\end{array}$ & $\begin{array}{r}107213 \\
4,2 \%\end{array}$ & $\begin{array}{r}126586 \\
4,3 \%\end{array}$ & $\begin{array}{r}129125 \\
4,4 \%\end{array}$ & $\begin{array}{r}140055 \\
4,2 \%\end{array}$ & $\begin{array}{r}159275 \\
4,5 \%\end{array}$ & $2,9 \%$ & $1,4 \%$ \\
\hline & $\begin{array}{l}\text { Centr. } \\
(10-100 \mathrm{k})\end{array}$ & $\begin{array}{r}321493 \\
17,4 \%\end{array}$ & $\begin{array}{r}455838 \\
17,9 \%\end{array}$ & $\begin{array}{r}529857 \\
18,1 \%\end{array}$ & $\begin{array}{r}550575 \\
18,6 \%\end{array}$ & $\begin{array}{r}635555 \\
19,0 \%\end{array}$ & $\begin{array}{r}635451 \\
17,8 \%\end{array}$ & $2,5 \%$ & $1,0 \%$ \\
\hline & $\begin{array}{l}\text { Péri. } \\
(10-100 \mathrm{k})\end{array}$ & $\begin{array}{r}254764 \\
13,8 \%\end{array}$ & $\begin{array}{r}355259 \\
13,9 \%\end{array}$ & $\begin{array}{r}384059 \\
13,1 \%\end{array}$ & $\begin{array}{r}391080 \\
13,2 \%\end{array}$ & $\begin{array}{r}417975 \\
12,5 \%\end{array}$ & $\begin{array}{r}402675 \\
11,3 \%\end{array}$ & $2,1 \%$ & $0,2 \%$ \\
\hline \multirow{8}{*}{$\begin{array}{l}\text { Secteur } \\
\text { Production } \\
\text { des biens }\end{array}$} & Prov. QC & $\begin{array}{r}582547 \\
31,6 \%\end{array}$ & $\begin{array}{r}694684 \\
27,2 \%\end{array}$ & $\begin{array}{r}637549 \\
21,8 \%\end{array}$ & $\begin{array}{r}622557 \\
21,0 \%\end{array}$ & $\begin{array}{r}703115 \\
21,1 \%\end{array}$ & $\begin{array}{r}666951 \\
18,7 \%\end{array}$ & $0,5 \%$ & $0,3 \%$ \\
\hline & Montréal & $\begin{array}{r}271730 \\
28,4 \%\end{array}$ & $\begin{array}{r}314726 \\
24,5 \%\end{array}$ & $\begin{array}{r}275380 \\
18,9 \%\end{array}$ & $\begin{array}{r}260885 \\
17,9 \%\end{array}$ & $\begin{array}{r}290535 \\
17,5 \%\end{array}$ & $\begin{array}{r}276560 \\
15,1 \%\end{array}$ & $0,1 \%$ & $0,4 \%$ \\
\hline & Québec & $\begin{array}{r}25082 \\
15,1 \%\end{array}$ & $\begin{array}{r}28904 \\
11,7 \%\end{array}$ & $\begin{array}{r}32370 \\
10,4 \%\end{array}$ & $\begin{array}{r}30820 \\
9,8 \%\end{array}$ & $\begin{array}{r}36805 \\
10,6 \%\end{array}$ & $\begin{array}{r}41055 \\
10,7 \%\end{array}$ & $1,3 \%$ & $1,9 \%$ \\
\hline & Sherb. & $\begin{array}{r}11913 \\
37,6 \%\end{array}$ & $\begin{array}{r}14757 \\
33,3 \%\end{array}$ & $\begin{array}{c}13749 \\
26,3 \%\end{array}$ & $\begin{array}{r}15369 \\
25,1 \%\end{array}$ & $\begin{array}{r}19225 \\
24,4 \%\end{array}$ & $\begin{array}{r}17350 \\
23,6 \%\end{array}$ & $0,7 \%$ & $0,8 \%$ \\
\hline & Tr.-Riv. & $\begin{array}{r}10965 \\
2 \%\end{array}$ & $\begin{array}{r}12688 \\
2 \%\end{array}$ & $\begin{array}{r}11498 \\
2 \%\end{array}$ & $\begin{array}{r}11508 \\
2 \%\end{array}$ & $\begin{array}{r}11745 \\
2 \%\end{array}$ & $\begin{array}{r}11675 \\
2 \%\end{array}$ & $0,2 \%$ & $0,1 \%$ \\
\hline & Saguenay & $\begin{array}{r}20828 \\
29,0 \%\end{array}$ & $\begin{array}{r}26770 \\
25,0 \%\end{array}$ & $\begin{array}{r}25308 \\
20,0 \%\end{array}$ & $\begin{array}{r}24273 \\
18,8 \%\end{array}$ & $\begin{array}{r}28545 \\
20,4 \%\end{array}$ & $\begin{array}{r}29460 \\
18,5 \%\end{array}$ & $1,0 \%$ & $1,3 \%$ \\
\hline & $\begin{array}{l}\text { Centr. } \\
(10-100 \mathrm{k})\end{array}$ & $\begin{array}{r}137189 \\
42,7 \%\end{array}$ & $\begin{array}{r}168040 \\
36,9 \%\end{array}$ & $\begin{array}{r}162538 \\
30,7 \%\end{array}$ & $\begin{array}{r}167072 \\
30,3 \%\end{array}$ & $\begin{array}{r}195145 \\
30,7 \%\end{array}$ & $\begin{array}{r}179883 \\
28,3 \%\end{array}$ & $0,9 \%$ & $0,5 \%$ \\
\hline & $\begin{array}{l}\text { Péri. } \\
(10-100 \mathrm{k})\end{array}$ & $\begin{array}{r}98393 \\
38,6 \%\end{array}$ & $\begin{array}{r}120585 \\
33,9 \%\end{array}$ & $\begin{array}{r}109012 \\
28,4 \%\end{array}$ & $\begin{array}{r}107089 \\
27,4 \%\end{array}$ & $\begin{array}{r}116885 \\
28,0 \%\end{array}$ & $\begin{array}{r}101495 \\
25,2 \%\end{array}$ & $0,5 \%$ & $-0,4 \%$ \\
\hline \multirow{8}{*}{$\begin{array}{l}\begin{array}{l}\text { Fonctions } \\
\text { haut } \\
\text { contenu } \\
\text { en savoir }\end{array} \\
\text { Secteur } \\
\text { Production } \\
\text { des biens }\end{array}$} & Prov. QC & $\begin{array}{r}50746 \\
8,7 \%\end{array}$ & $\begin{array}{r}68569 \\
9,9 \%\end{array}$ & $\begin{array}{r}82686 \\
13,0 \%\end{array}$ & $\begin{array}{r}95848 \\
15,4 \%\end{array}$ & $\begin{array}{r}121990 \\
17,3 \%\end{array}$ & $\begin{array}{r}130344 \\
19,5 \%\end{array}$ & $2,5 \%$ & $3,1 \%$ \\
\hline & Montréal & $\begin{array}{r}34618 \\
12,7 \%\end{array}$ & $\begin{array}{r}43054 \\
13,7 \%\end{array}$ & $\begin{array}{r}48131 \\
17,5 \%\end{array}$ & $\begin{array}{r}54697 \\
21,0 \%\end{array}$ & $\begin{array}{r}70255 \\
24,2 \%\end{array}$ & $\begin{array}{r}75000 \\
27,1 \%\end{array}$ & $1,7 \%$ & $2,1 \%$ \\
\hline & Québec & $\begin{array}{r}2062 \\
8,2 \%\end{array}$ & $\begin{array}{r}3383 \\
11,7 \%\end{array}$ & $\begin{array}{r}4884 \\
15,1 \%\end{array}$ & $\begin{array}{r}6083 \\
19,7 \%\end{array}$ & $\begin{array}{r}7755 \\
21,1 \%\end{array}$ & $\begin{array}{r}9425 \\
23,0 \%\end{array}$ & $4,4 \%$ & $3,0 \%$ \\
\hline & Sherb. & $\begin{array}{r}833 \\
4,8 \%\end{array}$ & $\begin{array}{l}1181 \\
6,6 \%\end{array}$ & $\begin{array}{r}1678 \\
10,2 \%\end{array}$ & $\begin{array}{r}2308 \\
11,1 \%\end{array}$ & $\begin{array}{r}3155 \\
13,2 \%\end{array}$ & $\begin{array}{r}3450 \\
14,1 \%\end{array}$ & $3,6 \%$ & $2,7 \%$ \\
\hline & Tr.-Riv. & $\begin{array}{r}600 \\
1 \%\end{array}$ & $\begin{array}{r}928 \\
1 \%\end{array}$ & $\begin{array}{r}1516 \\
2 \%\end{array}$ & $\begin{array}{r}1752 \\
2 \%\end{array}$ & $\begin{array}{r}1915 \\
2 \%\end{array}$ & $\begin{array}{r}2040 \\
2 \%\end{array}$ & $4,7 \%$ & $1,0 \%$ \\
\hline & Saguenay & $\begin{array}{l}1932 \\
9,3 \%\end{array}$ & $\begin{array}{r}3039 \\
11,4 \%\end{array}$ & $\begin{array}{r}3539 \\
14,0 \%\end{array}$ & $\begin{array}{r}3805 \\
15,7 \%\end{array}$ & $\begin{array}{r}4820 \\
16,9 \%\end{array}$ & $\begin{array}{r}5605 \\
19,0 \%\end{array}$ & $3,1 \%$ & $2,6 \%$ \\
\hline & $\begin{array}{l}\text { Centr. } \\
(10-100 \mathrm{k})\end{array}$ & $\begin{array}{r}6394 \\
4,7 \%\end{array}$ & $\begin{array}{r}9796 \\
5,8 \%\end{array}$ & $\begin{array}{r}13386 \\
8,2 \%\end{array}$ & $\begin{array}{r}16861 \\
10,1 \%\end{array}$ & $\begin{array}{r}21655 \\
11,1 \%\end{array}$ & $\begin{array}{r}23102 \\
12,8 \%\end{array}$ & $3,8 \%$ & $2,1 \%$ \\
\hline & $\begin{array}{l}\text { Péri. } \\
(10-100 \mathrm{k})\end{array}$ & $\begin{array}{r}4385 \\
4,5 \%\end{array}$ & $\begin{array}{r}7090 \\
5,9 \%\end{array}$ & $\begin{array}{r}9463 \\
8,7 \%\end{array}$ & $\begin{array}{r}10992 \\
10,3 \%\end{array}$ & $\begin{array}{r}13250 \\
11,3 \%\end{array}$ & $\begin{array}{r}11980 \\
11,8 \%\end{array}$ & $3,9 \%$ & $0,6 \%$ \\
\hline
\end{tabular}

Source: Statistique Canada, recensement de 1971-2006 - compilation spéciale LASER 
Annexe 2 Fonctions d'entreprises et caractéristiques socioéconomiques*, Canada 2006

\begin{tabular}{|c|c|c|c|c|}
\hline Fonction & $\begin{array}{l}\text { Équivalent } \\
\text { CTP } 2006\end{array}$ & Groupe professionnel principal & $\begin{array}{c}\text { \% diplôme égal } \\
\text { ou supérieur au } \\
\text { B.A, B.Sc }\end{array}$ & $\begin{array}{c}\text { Revenu } \\
\text { moyen } \\
\text { annuel }\end{array}$ \\
\hline Gestion & A, B0, B1, B3 & $\begin{array}{l}\text { Gérants, directeurs } \\
\text { et professions apparentées }\end{array}$ & $34,7 \%$ & $72112 \$$ \\
\hline Scientifiques / Professionnels & $\begin{array}{l}\text { C, E0, E211, } \\
\text { E212, E213 }\end{array}$ & $\begin{array}{l}\text { Sciences naturelles, ingénierie, } \\
\text { mathématique; sciences sociales }\end{array}$ & $50,5 \%$ & $65155 \$$ \\
\hline Haut contenu en savoir & --- & -- & $40,8 \%$ & $69435 \$$ \\
\hline Éducation/Santé* & $\begin{array}{l}D, E 1, E 214, \\
E 215, E 216, F\end{array}$ & Religion, éduc., santé, art, loisirs & $49,1 \%$ & $54124 \$$ \\
\hline Soutien administratif & $\mathrm{B} 2, \mathrm{~B} 4, \mathrm{~B} 5$ & Administration et autres professions & $13,3 \%$ & $37400 \$$ \\
\hline Vente et services & G & Vente et services & $9,4 \%$ & $35830 \$$ \\
\hline Production / Entretien & $\mathrm{H}, \mathrm{I}, \mathrm{J}$ & $\begin{array}{l}\text { Agriculture, pêche; } \\
\text { expl. forestière, mines. }\end{array}$ & $4,7 \%$ & $42500 \$$ \\
\hline Manuel-Routine & -- & --- & $15,7 \%$ & $41662 \$$ \\
\hline
\end{tabular}

Source: Statistique Canada. Recensement de 2006. Catalogue no 97-564-ХCB2006005

\section{Annexe 3 Secteur de la production des biens et sous-industries}

\begin{tabular}{|c|c|c|c|}
\hline & Industrie & Code SCIAN & Principales industries \\
\hline \multirow{5}{*}{$\begin{array}{l}\text { Secteur de la } \\
\text { production des biens }\end{array}$} & Extraction primaire & 11,21 & $\begin{array}{l}\text { Agriculture, forêt, } \\
\text { mines, gaz et pétrole }\end{array}$ \\
\hline & $\begin{array}{l}\text { Manufacturiers de basse } \\
\text { technologie }\end{array}$ & $\begin{array}{l}311,312,316,313 \\
314,315,3262,337\end{array}$ & $\begin{array}{l}\text { Nourriture, textile, } \\
\text { vêtement et meuble }\end{array}$ \\
\hline & Première transformation & $\begin{array}{l}321,322,331,324 \\
327\end{array}$ & $\begin{array}{l}\text { Bois, papier, } \\
\text { métaux primaires et minéraux }\end{array}$ \\
\hline & $\begin{array}{l}\text { Manufacturiers de moyenne } \\
\text { technologie }\end{array}$ & $323,3261,332,333$ & $\begin{array}{l}\text { Impression, plastiques, } \\
\text { produits métalliques et machinerie }\end{array}$ \\
\hline & $\begin{array}{l}\text { Manufacturiers de haute } \\
\text { technologie }\end{array}$ & $334,335,336,339$ & $\begin{array}{l}\text { Transport, produits } \\
\text { électriques, électroniques et chimie }\end{array}$ \\
\hline
\end{tabular}

\section{Annexe 4 Caractéristiques du système urbain québécois, villes et régions synthétiques}

\begin{tabular}{lcccc}
\hline $\begin{array}{l}\text { Villes et régions } \\
\text { synthétiques }\end{array}$ & $\begin{array}{l}\text { Population } \\
(\mathbf{x 1 0 0 0})\end{array}$ & $\begin{array}{l}\text { Distance réticulaire } \\
\text { d'une région } \\
\text { métropolitaine } \\
\text { (temps de transport) }\end{array}$ & $\begin{array}{l}\text { Nombre d'unités } \\
\text { spatiales 1971-2001 } \\
\text { (Québec=95) }\end{array}$ & $\begin{array}{l}\text { Nombre d'unités } \\
\text { spatiales pour 2006 } \\
\text { (Québec=91) }\end{array}$ \\
\hline $\begin{array}{l}\text { Montréal } \\
\text { Québec }\end{array}$ & 3636 & 0 & 1 & 1 \\
$\begin{array}{l}\text { Sherbrooke } \\
\text { Trois-Rivières }\end{array}$ & 187 & Moins de 90 minutes & 1 & 1 \\
Saguenay & 142 & Moins de 90 minutes & 1 & 1 \\
Centrales (10k-100k) & 152 & Plus de 90 minutes & 1 & 1 \\
Périphériques (10k-100k) & $10-100$ & Moins de 90 minutes & 46 & 1 \\
\hline
\end{tabular}




\section{Annexe 5 Équations}

1) Déviation de la moyenne (DM) : Fonctions - Indicateur de la spécialisation fonctionnelle

$$
D M_{o i j}=R_{o i j}-R_{o i \bullet}
$$

Où

$$
\begin{aligned}
D M_{o i j}= & \text { déviation de la moyenne }(\mathrm{DM})-\text { Ratio emploi Fonction } o \\
& \text { (haut contenu) / Routine-production, pour l'industrie } i \text {, dans la région } j \\
R_{o i j}= & \text { ratio d'emploi Fonction } o \text { (haut contenu) / Routine-production, } \\
& \text { pour l'industrie } i \text {, dans la région } j
\end{aligned}
$$

2) Quotient de localisation: Secteur de la production des biens -

Indicateur de la spéciation sectorielle

$$
L Q_{i j}=P_{i j} / P_{i \bullet}
$$

Où

$$
\begin{aligned}
L Q_{i j} \quad= & \text { quotient de localisation de l'industrie } i \text { (secteur de la production } \\
& \text { des biens), dans la région } j
\end{aligned}
$$

3) Taux de croissance annuel moyen (TCAM), fonctions à haut contenu en savoir, secteur de la production des biens

$$
T C A M_{o i j, t-t-1}=\left(\sqrt[n]{\frac{E_{o i j, t}}{E_{o i j, t-1}}-1}\right) \times 100
$$

Où

$$
\begin{aligned}
& T C A M_{o i j}=\text { taux de croissance annuel moyen, fonctions à haut contenu en savoir } \\
& o \text {, dans l'industrie } i \text { (secteur de la production des biens), dans la } \\
& \text { région } j \text {, entre la période initiale } t-1 \text { et finale } t \\
& E_{o i j, t} \quad=\text { emploi dans la fonction } o \text { (haut contenu), dans l'industrie } i \text { (secteur } \\
& \text { de la production des biens), dans la région } j \text {, à la période finale } t \\
& E_{o i j, t-1}=\text { emploi dans la fonction } o \text { (haut contenu), dans l'industrie } i \text { (secteur } \\
& \text { de la production des biens), dans la région } j \text {, à la période initiale } t-1 \\
& \mathrm{n}=\text { nombre d'années entre la période finale } t \text { et la période initiale } t-1
\end{aligned}
$$




\section{Bibliographie}

AARLAND, Kristin, DAVIS, James C., HENDERSON, J. Vernon et ONO, Yukako (2007) Spatial organization of firms: The decision to split production and administration. RAND Journal of Economics, vol. 38, p. 480-494.

APPARICIO, Philippe, POLÈSE, Mario et SHEARMUR, Richard (2009) Une histoire de résidus: à propos des facteurs généraux et locaux de croissance régionale au Canada, de 1971 à 2001. Le Géographe canadien, vol. 53, nº 4, p. 385-403.

AYDALOT, Philippe (1976) Dynamique spatiale et développement inégal. Paris, Economica.

BADE, Franz-Josef (1983) Large corporations and regional development. Regional Studies, vol. 17, p. 315-325.

BADE, Franz-Josef, LAASER, Claus-Friedrich et SOLTWEDEL, Rüdiger (2004) Urban specialization in the Internet age - Empirical findings for Germany. Kiel Working Papers. Kiel, Kiel Institute for the World Economy, p. 32.

BALDWIN, Richard (2009) Integration of the North American economy and newparadigm globalization. Economic Research and Analysis. Ottawa, Government of Canada - Policy Research Initiative.

BARBOUR, Elisa et MARKUSEN, Ann (2007) Regional occupational and industrial structure: Does one imply the other? International Regional Science Review, vol. 30, p. 72-90.

BOSCHMA, Ron et IAMMARINO, Simona (2009) Related variety, trade linkages, and regional growth in Italy. Economic Geography, vol. 85, p. 289-311.

BROWN, Shannon (2008) Business processes and business functions; a new way of looking at employment. Monthly Labor Review, p. 51-70.
BRUNELLE, Cédric et POLÈSE, Mario (2008) Functional specialization across space: A case study of the Canadian electricity industry, 1971-2001. Canadian Geographer, vol. 52, no 4 , p. 486-504.

CHANDLER, Alfred D. (1977) The visible hand: The managerial revolution in American business. Cambridge, Harvard University Press.

DAVIS, James C. et HENDERSON, J. Vernon (2008) The agglomeration of headquarters. Regional Science and Urban Economics, n³8, p. 445-60.

DEAN, Judith, FUNG, K.C. et WANG, Zhi (2007) Measuring the vertical specialization in Chinese trade. U.S. International Trade Commission.

DEFEVER, Fabrice (2005) Décomposition internationale de la chaîne de valeur: une étude de la localisation des firmes multinationales dans l'Europe élargie. Revue Économique, n56, p. 1185-1205.

DEFEVER, Fabrice (2006) Functional fragmentation and the location of multinational firms in the enlarged Europe. Regional Science and Urban Economics, nº36, p. 658-677.

DOLOREUX, David, FILION, Pierre et KLEIN, Juan-Luis (2005) Systèmes régionaux et innovation: le cas de la Beauce québécoise. Dans Régis Guillaume (dir.) Globalisation, systèmes productifs et dynamiques territoriales. Regard croisés au Québec et dans le Sud-ouest français. Paris, L'Harmattan.

DOLOREUX, David, DIONNE, Stève et JEAN, Bruno (2007) The evolution of an innovation system in a rural area: The case of La Pocatière, Québec. International Journal of Urban and Regional Research, no31, p. 146-167.

DOLOREUX, David et MELANÇON, Yannik (2008) On the dynamics of innovation in Quebec's coastal maritime industry. Technovation, n²8, p. 231-143. 
DURANTON, Gilles et PUGA, Diego (2005) From sectoral to functional urban specialisation. Journal of Urban Economics, no57, p. 343-370.

FUJITA, Masahisa et THISSE, JacquesFrancois (2006) Globalization and the evolution of the supply chain: Who gains and who loses? International Economic Review, nº47, p. 811-836.

GREENE, William H. (2005) Econometric analysis. Prentice Hall, New Jersey.

HELSLEY, Robert W. et STRANGE, William C. (2007) Agglomeration, opportunism, and the organization of production. Journal of Urban Economics, nº62, p. 55-75.

HENDERSON, Vernon, SHALIZI, Zmarak et VENABLES, Anthony J. (2001) Geography and development. Journal of Economic Geography, no 1, p. 81-105.

HENDRICKS, Lutz (2011) The skill composition of US Cities. International Economic Review, no52, p. 1-32.

HUMMELS, David, ISHII, Jun et YI, Kei-Mu (2001) The nature and growth of vertical specialization in world trade. Journal of International Economics, nº 54, p. 75-96.

HUWS, Ursula, DAHLMANN, Simone, FLECKER, Jörg, HOLTGREWE, Ursula, SCHÖNAUER, Annika, RAMIOUL, Monique et GEURTS, Karen (2009) Value chain restructuring in Europe in a global economy. Dans Ursula Huws (dir.) Work organisation and restructuring in the knowledge society. Katholieke Universiteit Leuven: Higher Institute of Labour Studies, Leuven, p. 111.

JULIEN, Pierre-André (1997) Le développement régional. Comment multiplier les Beauce au Québec. Québec, Les éditions de l'Institut québécois de recherche sur la culture.

JULIEN, Pierre-André (2005) Entrepreneuriat régional et économie de la connaissance. Québec, Presses de l'Université du Québec.

KOO, Jun (2005) How to analyze the regional economy with occupation data. Economic Development Quarterly, nº19, p. 356-372.
LEAMER, Edward E. et STORPER, Michael (2001) The economic geography of the Internet age. Journal of International Business Studies, no32, p. 641-665.

LEMELIN, André (2008) Montréal dans l'économie du Québec. Montréal, Centre Urbanisation, Culture et Société de l'INRS.

LOBO E SILVA, Carlos Eduardo et HEWINGS, Geoffrey J. D. (2010) The locational implications of management and production fragmentation. Estudos Economicos, n ${ }^{\circ}$, p. 515-533.

MASSEY, Doreen (1984) Spatial divisions of labour: Social structures and the geography of production. Londres, Macmillan.

MORETTI, Enrico (2010) Local multipliers. American Economic Review, no 100, p. 373-377.

OCDE (2007) Comment rester compétitif dans l'économie mondiale: progresser dans la chaîne de valeur. Rapport de synthèse, Paris.

ONO, Yukako (2003) Outsourcing business services and the role of central administrative offices. Journal of Urban Economics, no53, p. 377-395.

POLÈSE, Mario, SHEARMUR, Richard, DESJARDINS, Pierre-Marcel et JOHNSON, Marc (2002) La périphérie face à l'économie du savoir: la dynamique spatiale de l'économie canadienne et l'avenir des régions non métropolitaines du Québec et des provinces de l'Atlantique. Montréal, Centre Urbanisation, Culture et Société de l'INRS et Institut canadien de recherche sur le développement régional.

POLĖSE, Mario et SHEARMUR, Richard (2005) Why some regions will decline: A Canadian case study with thoughts on local development strategies. Papers in Regional Science, no 85, p. 23-46.

POLĖSE, Mario et SHEARMUR, Richard (2006) Growth and location of economic activity: The spatial dynamics of industries in Canada 1971-2001. Growth and Change, no37, p. 362-395. 
POLÈSE, Mario (2009) Les nouvelles dynamiques régionales de l'économie québécoise: cinq tendances. Recherches sociographiques, n50, p. 11-40.

POLÈSE, Mario (2012) L'autre «mystère de Québec». Regards sur une mutation économique étonnante. Recherches sociographiques, no53, p. 133-156.

PRED, Allan R. (1974) Major job-providing organizations and systems of cities. Commission on College Geography. Association of American Geographers, Washington, D.C.

PRED, Allan R. (1975) On the spatial structure of organizations and the complexity of metropolitan interdependence. Papers of the Regional Science Association, no35, p. 115-142.

PROULX, Marc-Urbain (2009) Territoires émergents et cohérence dans l'espace Québec. Cahiers de géographie du Québec, vol. 53,no 149 , p. 177-196. [En ligne]. http://www.erudit.org/revue/cgq/2009/ v53/n149/038781ar.pdf

ROSSI-HANSBERG, Esteban, SARTE, PierreDaniel et OWENS III, Raymond (2009) Firm fragmentation and urban patterns. International Economic Review, n ${ }^{50}$, p. 143-186.

SALLEZ, Alain (1983) Division spatiale du travail, développement régional polarisé et théorie de la localisation. Revue d'Économie Régionale et Urbaine, $\mathrm{n}^{\circ}$ 1, p. 69-96.

SCOTT, Allen J. (2010) Space-time variations of human capital assets across U.S. metropolitan areas, 1980 to 2000. Economic Geography, no 86 , p. 233-249.

SHEARMUR, Richard et POLÈSE, Mario (2005) Diversity and employment growth in Canada, 1971-2001: Can diversification policies succeed? Canadian Geographer, vol. 49, n³ 3 , p. 272-290.

STOCK, James H. et WATSON, Mark W. (2007) Introduction to econometrics. Boston, Pearson.

STORPER, Michael (2010) Why does a city grow? Specialisation, human capital or institutions? Urban Studies, vol. 47, p. 2027-2050.
STURGEON, Timothy et GEREFFI, Gary (2008) The challenge of global value chains: Why integrative trade requires new thinking and new data. Industrie Canada, Ottawa.

STURGEON, Timothy et GEREFFI, Gary (2009) Measuring success in the global economy: International trade, industrial upgrading, and business function outsourcing in global value chains. Transnational Corporations, $\mathrm{n}^{\circ} 18$, p. 1-36.

THOMPSON, Wilbur R. (1986) Cities in transition. The Annals of the Political and Social American Academy of Science, n० 488 , p. 18-34.

THOMPSON, Wilbur R. et THOMPSON, Philip R. (1987) National industries and local occupational strengths: The crosshairs of targeting. Urban Studies, no 24 , p. 547-560.

WESTAWAY, John (1974) The spatial hierarchy of business organizations and its implications for the British urban system. Regional Studies, nº 8, p. 145-155.

WOOLDRIDGE, Jeffrey M. (2002) Econometric analysis of cross section and panel data. Cambridge, MIT University Press. 